\title{
Elusiveness of evidence for multifragmentation in 1-GeV proton-nucleus reactions
}

\author{
Davide Mancusi, ${ }^{1, *}$ Alain Boudard, ${ }^{2}$ Joseph Cugnon, ${ }^{1}$ Jean-Christophe David, ${ }^{2}$ Thomas Gorbinet, ${ }^{2}$ and Sylvie Leray ${ }^{2}$ \\ ${ }^{1}$ Fundamental Interactions in Physics and Astrophysics, University of Liège, allée du 6 août 17, bât. B5, B-4000 Liège 1, Belgium \\ ${ }^{2}$ CEA, Centre de Saclay, IRFU/Service de Physique Nucléaire, F-91191 Gif-sur-Yvette, France
}

(Received 28 October 2011; published 27 December 2011)

\begin{abstract}
We use the tools of hybrid intranuclear-cascade/nuclear-deexcitation models to evaluate the sensitivity of several physical observables to the inclusion of a multifragmentation stage in the deexcitation chain and assess the need for a multifragmentation model in the quantitative description of $p+{ }^{56} \mathrm{Fe}$ and $p+{ }^{136} \mathrm{Xe}$ reactions at $1-\mathrm{GeV}$ incident energy. We seek clear signatures of multifragmentation by comparing different state-of-the-art deexcitation models coupled with intranuclear-cascade models and by focusing on discriminating observables such as correlations and fragment longitudinal-velocity distributions. None of the considered observables can be unambiguously interpreted as a multifragmentation footprint. The experimental data are best described as originating from sequential binary decays. However, no deexcitation model can reproduce the experimental longitudinal-velocity distributions from $1-\mathrm{GeV} p+{ }^{136} \mathrm{Xe}$.
\end{abstract}

DOI: 10.1103/PhysRevC.84.064615

PACS number(s): 24.10.Lx, 25.40.Sc, 25.70.Mn, 25.70.Pq

\section{INTRODUCTION}

Multifragmentation is generally considered the quasisimultaneous breakup of highly excited nuclear matter into clusters and unbound nucleons. Interest in this phenomenon was first triggered by anomalously large production cross sections of intermediate-mass fragments (abbreviated as IMFs and defined as $3 \leqslant Z \leqslant 10$ for the purposes of this paper) from intermediate-energy heavy-ion collisions (see Ref. [1] for a collection of recent reviews). The earliest theoretical explanations suggested the interpretation whereby the typical power-law distribution of fragment masses is a signature of liquid-vapor equilibrium of nuclear matter near the critical temperature. In this scheme, nuclear clusters are formed within a short time span, with large multiplicities, as condensation droplets of a vapor of nucleons. However, as the initial enthusiasm over liquid-vapor multifragmentation faded and other candidate models (e.g., statistical multifragmentation, spinodal instability, and even sequential binary decays) were put forward to explain the data, it was quickly realized that the power-law signature was by no means unique to liquid-vapor multifragmentation. Remarkably, even simple percolation models are able to reproduce most of the features of the observed IMF distributions. Therefore, other observables must be sought if one wishes to discriminate among the proposed IMF production mechanisms, which are, in any case, not necessarily mutually exclusive.

One of the main difficulties of multifragmentation studies based on heavy-ion reactions is that there is considerable theoretical uncertainty on the reaction dynamics and on the importance of collective effects such as deformation or compression. In nucleon-induced reactions, on the other hand, it is difficult to imagine that the collective state of the system can be strongly perturbed. Since it had been known for a long

\footnotetext{
*Present address: CEA, Centre de Saclay, IRFU/Service de Physique Nucléaire, F-91191 Gif-sur-Yvette, France; davide.mancusi@cea.fr
}

time that IMFs could also be produced in nucleon-induced reactions, multifragmentation studies were also performed on these better-understood systems, although the excitation energies that can be reached by this method are typically lower.

Today, the importance of multifragmentation in nucleoninduced reactions is the subject of long-standing discussion. While it is generally accepted that multifragmentation will eventually set in at high projectile energy, due to the increasing energy transfer from the projectile to the target nucleus, it is not yet clear whether and to what extent multifragmentation needs to be postulated for a reliable quantitative description of reactions around $1 \mathrm{GeV}$, a region which is most interesting for technical applications such as accelerator-driven systems (ADS) [2], radioprotection in space [3], and shielding at accelerators [4].

The recent IAEA-promoted "Benchmark of Spallation Models," which focused on the 60- to 3000-MeV incidentenergy range, represents an effort "to assess the prediction capabilities of the spallation models used or that could be used in the future in high-energy transport codes; to understand the reason for the success or deficiency of the models in the different mass and energy regions or for the different exit channels; to reach a consensus, if possible, on some of the physics ingredients that should be used in the models" [5]. The benchmark saw the participation of 17 spallation models, all of which were couplings of a dynamical reaction model (intranuclear cascade, quantum molecular dynamics...) and a statistical-decay model, with the possible presence of an intermediate pre-equilibrium stage. Since not all the participating models include a multifragmentation stage, it is, in principle, possible to study the benchmark results and estimate the sensitivity of the benchmark end points (isotopic production cross sections, excitation curves, neutron-multiplicity distributions, and double-differential cross sections for neutrons, light-charged particles, and pions) to the multifragmentation process. In particular, by comparing the predictions of different deexcitation models coupled with a fixed dynamical stage, one can extract precious information about the influence of deexcitation alone. 
However, previous studies have already indicated that inclusive observables, such as double-differential nucleon spectra or nuclide yields, are rather insensitive to the inclusion of a multifragmentation stage in the deexcitation chain [6]. Hence, characteristic signatures of multifragmentation must be sought among other, more discriminating observables. The impact of a multifragmentation stage in the deexcitation chain can, in principle, be assessed by comparing calculation results with experimental data.

The goal of the present work is to identify possible signatures of multifragmentation by studying nucleoninduced reactions with the tools of coupled intranuclearcascade/deexcitation models. We shall focus on the $1-\mathrm{GeV}$ $p+{ }^{56} \mathrm{Fe}$ and $p+{ }^{136} \mathrm{Xe}$ reactions, which have been the object of recent studies [6-10]. The small mass of the $p+{ }^{56} \mathrm{Fe}$ system leads to the production of a limited number of nuclides. Several deexcitation mechanisms can contribute to a given nuclide yield, making it more difficult to extract an unambiguous multifragmentation signature from a background of deexcitation residues and/or direct IMF emissions. On the other hand, the multifragmentation threshold may be more easily attained in $p+{ }^{56} \mathrm{Fe}$, which realizes higher excitation energies per nucleon. Thus, the two systems studied are complementary. Heavier systems are excluded from the present study, in order to avoid the conceptual and technical complications connected to the competition between light-fragment emission and fission.

The paper is organized as follows: In Sec. II we give a brief overview of the models used for the study. Section III examines the sensitivity of the considered observables to the choice of the intranuclear-cascade model. Section IV discusses the available inclusive residue-production data and how they are reproduced by the different deexcitation models. Section V presents the model predictions for the SPALADIN correlation data set for $p+{ }^{56} \mathrm{Fe}$ [6]. Section VI discusses longitudinalvelocity distributions measured in inverse kinematics at GSI (Darmstadt, Germany) [9,10]. Section VII discusses time intervals between fragment emissions in INCL4.5/GEMINI++. Finally, Sec. VIII summarizes our conclusions.

\section{MODEL OVERVIEW}

All the calculations presented in this paper were performed using a coupled intranuclear-cascade/statistical-deexcitation model. We used two intranuclear-cascade models (INCL4.5 and ISABEL) coupled with three different deexcitation models (ABLA07, GEMINI++, and SMM). Since the focus of this paper is on deexcitation, we will limit ourselves to directing the reader to the relevant publications for details about the physics of the cascade models.

\section{A. Cascade models}

The Liège Intranuclear Cascade model (INCL) [11,12] is one of the most refined existing tools for the description of nucleon-, pion- and light-ion-induced reactions in the 150 to $3000-\mathrm{MeV}$ incident energy range. The model is currently maintained and developed jointly by the University of Liège (Liège, Belgium) and CEA (Saclay, France). It can describe the emission of nucleons and pions; light clusters (up to $Z=5$,
$A=8$ with the default program options) can also be produced through a dynamical phase-space coalescence algorithm. The INCL model is not to be considered as adjustable. It does contain parameters, but they are either taken from known phenomenology (such as the matter density radius of the nuclei) or have been adjusted once for all (such as the parameters of the Pauli blocking or those that determine the coalescence module for the production of the light-charged clusters). The predictions of INCL concerning those observables that can be confronted directly to experiment, namely the high-energy parts of particle spectra, are of rather good quality, as recently shown [12]. The INCL/ABLA07, INCL/GEMINI++, and INCL/SMM combinations were also recognized among the best-performing participants of the IAEA "Benchmark of Spallation Models" [5]. The present work is based on the INCL version that was used for the IAEA benchmark, plus some minor bug fixes; this version is known as INCL4.5.

An older version of the INCL model, known as INCL4.2, was employed for studying the SPALADIN correlation data set [6]. The most important differences between INCL4.2 and 4.5 are reviewed in Ref. [12] and include the introduction of the cluster-coalescence algorithm, energy- and isospin-dependent potentials for nucleons and pion potentials, as well as an improvement of Pauli blocking. More details are given in the reference above.

The ISABEL model [13], no longer developed, has vastly contributed to the understanding of nucleon-nucleus and nucleus-nucleus reactions. It is mainly used in the present work to highlight the sensitivity of the studied observables to cascade.

\section{B. Deexcitation models}

\section{ABLA07}

The ABLA07 model [14] is maintained and developed by the CHARMS group at GSI (Darmstadt, Germany). The model contains a multifragmentation submodule, which is triggered only if the temperature of the compound nucleus exceeds a mass-dependent (as suggested by Natowitz et al. [15]) freezeout threshold:

$$
T_{\text {freeze-out }}(A)=\max \left[5.5,9.337 \exp \left(-2.82 \times 10^{-3} A\right)\right] \mathrm{MeV} \text {. }
$$

In that case, the system breaks up into fragments whose mass is distributed according to an empirical power-law spectrum and whose momenta carry Goldhaber-type and thermal contributions. Coulomb repulsion among multifragmentation products is accounted for in a simplified manner. The excitation energies of the resulting fragments are determined by assuming thermal equilibrium at the freeze-out temperature. Subsequent deexcitation of the multifragmentation products is assumed to be purely binary. If the multifragmentation module is not triggered, the initial compound nucleus directly enters the secondary de-excitation phase.

During secondary deexcitation, emission of any stable nucleus up to half the mass of the compound nucleus is possible, and it is quantitatively described by the Weisskopf-Ewing evaporation formalism [16]. Above the Businaro-Gallone point, competition with fission is treated dynamically and 
it is based on solutions of the Fokker-Planck equation for collective deformation of the nucleus over the fission barrier. ABLA07's fission module is among the most sophisticated models available on the market, but it is only of marginal interest for the systems studied in this paper.

Finally, subsequent binary decays are assumed to be independent; in particular, Coulomb interactions among particles produced in different decays are neglected. This assumption is customary in binary deexcitation models.

A less-sophisticated version of the ABLA model was considered for the study of the SPALADIN correlations [6] and was found unable to reproduce the measured residueproduction cross sections in $p+{ }^{56} \mathrm{Fe}$. It included neither multifragmentation nor evaporation of fragments heavier than $\alpha$ particles. More details about the differences between the two versions can be found in Ref. [14].

\section{GEMINI++}

The GEMINI++ model, developed by R. J. Charity [17], represents an effort to describe nuclear deexcitation uniquely in terms of binary decays. No simultaneous breakup is allowed. Multifragment events can, of course, be produced by sequences of binary fragment emissions; as in the case of ABLA07, Coulomb interactions among particles emitted in different decays are neglected. Emission of light particles $(Z \leqslant 3$ by default) is described by the Hauser-Feshbach evaporation formalism [18]; Moretto's conditional-saddle-point formalism [19] with Sierk's finite-range barriers [20] is used for complexfragment emission. For heavy systems, the fission width is calculated using a refined Bohr-Wheeler approach [21].

GEMINI++'s asymmetric-fission module has recently been improved [22] to describe fragment yields from fusion and spallation reactions with the same parameter set. To this end, it was necessary to augment Sierk's barriers by a constant shift of $7 \mathrm{MeV}$, which can be interpreted as the difference in Wigner energy between the mother nucleus and the nascent fragments. However, this interpretation is not devoid of complications; see the relevant papers for more details.

\section{SMM}

The statistical multifragmentation model (SMM) [23,24], presently maintained by A. S. Botvina, is one of the most successful and widely applied multifragmentation models. Like in ABLA07 the first possible decay stage is the simultaneous breakup of the thermalized source in a number of hot fragments and particles. Unlike ABLA07's semiempirical approach, SMM always enters this submodule and samples breakup configurations according to their thermodynamical weight in a given freeze-out volume. The ratio of the freeze-out volume to the saturation volume is a free parameter and has been taken equal to 3 for both systems studied, which is also the value recommended by the authors of the model. With this parameter choice, single-fragment configurations (i.e., compound nucleus) naturally dominate the multifragmentation stage at low excitation energy; the importance of multifragment configurations smoothly increases and starts dominating the thermodynamical weight around $3 A \mathrm{MeV}$ excitation energy. Mass, charge, excitation energy, and momentum of the emerging hot fragments are sampled respecting conservation laws. Coulomb acceleration is then accounted for by solving the Hamilton equations for the propagation of the fragments in their mutual Coulomb field.

Secondary deexcitation is then applied to the hot fragments. If they are sufficiently light $(A \leqslant 16)$, the Fermi breakup model is applied. Otherwise, according to a modified WeisskopfEwing [16] scheme, they can evaporate particles up to ${ }^{18} \mathrm{O}$. Fission is described by the Bohr-Wheeler model [25].

Note that the SMM version used for the present work employs slightly different evaporation barriers compared to the IAEA benchmark. Barriers are computed using the standard formula

$$
B=(1.44 \mathrm{MeVfm}) \frac{Z_{1} Z_{2}}{r_{0}\left(A_{1}^{1 / 3}+A_{2}^{1 / 3}\right)} .
$$

In the IAEA benchmark, $r_{0}=1.5 \mathrm{fm}$ was used. In the present work, $r_{0}$ is determined as

$$
r_{0}=2.173 \cdot \frac{1+6.103 \times 10^{-3} Z_{1} Z_{2}}{1+9.443 \times 10^{-3} Z_{1} Z_{2}} \mathrm{fm} .
$$

This difference is marginal as far as IMF cross sections are concerned.

\section{BASIC CASCADE RESULTS}

As a first, basic comparison, Fig. 1 shows distributions of excitation energy and mass loss of cascade remnants of $1-\mathrm{GeV}$ $p+{ }^{56} \mathrm{Fe}$ and $p+{ }^{136} \mathrm{Xe}$. Already at this stage, it is possible to observe that INCL4.5 and ISABEL are not equivalent. INCL4.5 produces, on average, hotter and lighter remnants than ISABEL, although differences in excitation energy are smaller for ${ }^{136} \mathrm{Xe}$ than for ${ }^{56} \mathrm{Fe}$, as quantified in Tables I and II. Note that the reaction cross sections predicted by the two codes differ by only a few percentage points; thus, differences in the remnant characteristics must trace back to different cascade histories.

Previous investigations had found that INCL's and ISABEL's excitation-energy distributions for $p+{ }^{56} \mathrm{Fe}$ were remarkably similar [6]. The claim concerned version 4.2 of the INCL code [11], which is represented in Fig. 1 by the blue lines. This state of affairs was evidently modified by later developments of the INCL code [12]. We stress that the similarity between INCL4.2 and ISABEL's results can at least partly be explained by the very similar physics content of the models. Excitation energies are also sensibly larger in INCL4.5 than in INCL4.2. However, this difference cannot be simply ascribed to a single cause but rather represents the combined effect of several new physics ingredients, such as energy-dependent nucleon potentials and pion potentials.

One major difference between ISABEL and INCL4.5 (and between INCL4.2 and INCL4.5) is INCL4.5's ability to dynamically produce light-charged composite particles (see Sec. II A). Figure 1 depicts INCL4.5's predictions with (solid black lines) and without (dotted black lines) cluster coalescence. One immediately observes that ISABEL's mass distributions are remarkably similar to those predicted by INCL4.5 without coalescence. Clearly, INCL4.5's cluster emission algorithm reduces the remnant mass but does not sensibly affect the excitation-energy distribution. This is quite well understood: 

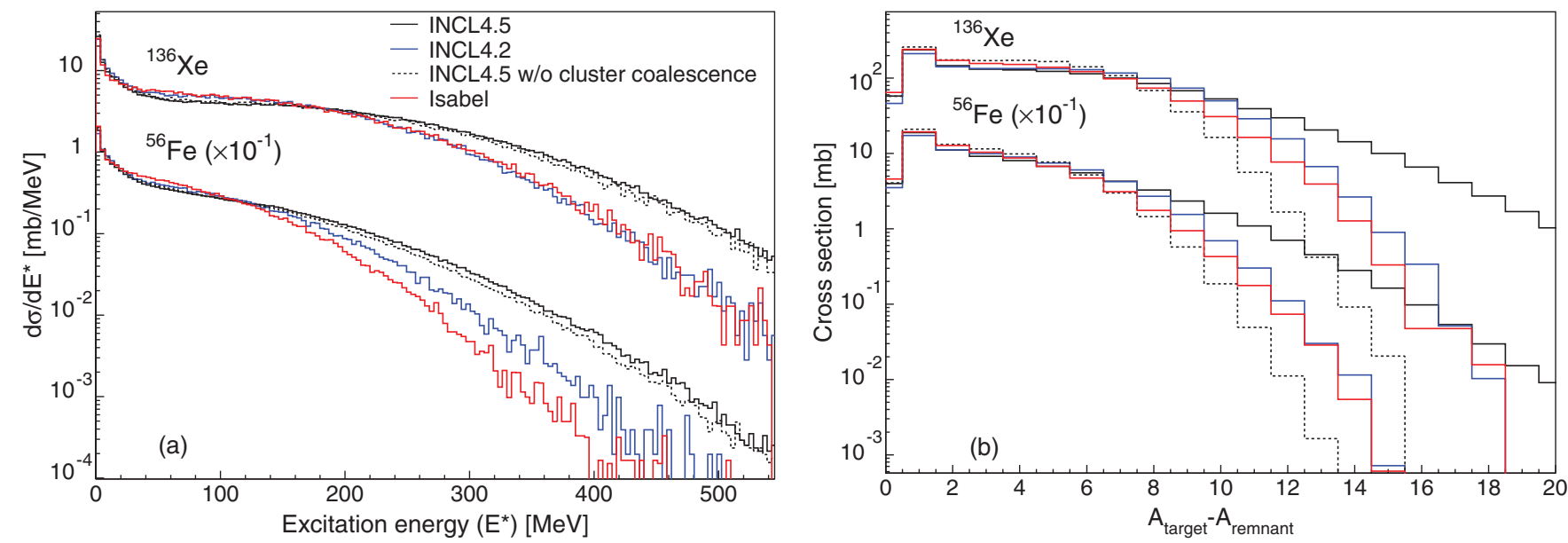

FIG. 1. (Color online) Comparison of excitation-energy (a) and mass-loss (b) distributions for remnants of the $1-\mathrm{GeV} p+{ }^{56} \mathrm{Fe}$ and $p+{ }^{136} \mathrm{Xe}$ reactions, as calculated by different cascade models.

Escaping clusters typically extract spectator nucleons close to the Fermi sea, thereby reducing the mass of the remnant but without significantly affecting its excitation energy. This result is also consistent with the documented behavior of an older, less-refined clustering algorithm [26].

Therefore, we conclude this section by observing that, contrary to what was claimed in Ref. [6], the choice of the intranuclear-cascade model does have some importance. In what follows, we shall discuss how the differences in remnant distributions are reflected in the residue-production cross sections and how these observables can guide us in selecting the cascade model that should be used for the study of more discriminating observables.

\section{RESIDUE-PRODUCTION CROSS SECTIONS}

Figures 2 and 3 show the predicted residue-production cross sections as a function of the nuclide charge, compared with the measurements obtained with the SPALADIN apparatus [6] or at the Fragment Separator (FRS) [7-9]. The two experiments have different acceptance cuts. The SPALADIN data, which will be discussed in detail in Sec. V, present a kinematical cut on particles with large longitudinal velocities with respect to the initial ${ }^{56} \mathrm{Fe}$ nucleus, which are mostly nucleons and light particles. The acceptance was estimated to be virtually complete for $Z \geqslant 4$. In the FRS data, on the other hand, only

TABLE I. Reaction cross section, average remnant excitation energies (total and per nucleon), and average remnant mass predicted by INCL4.5, ISABEL, INCL4.5 without cluster coalescence, and INCL4.2 for $1-\mathrm{GeV}$ proton-induced reactions on ${ }^{56} \mathrm{Fe}$.

\begin{tabular}{lcccc}
\hline \hline & INCL4.5 & ISABEL & $\begin{array}{c}\text { INCL4.5 } \\
\text { (no clusters) }\end{array}$ & INCL4.2 \\
\hline$\sigma^{\text {reac }}(\mathrm{mb})$ & 779 & 740 & 779 & 742 \\
$\left\langle E^{*}\right\rangle(\mathrm{MeV})$ & 91.5 & 66.6 & 85.9 & 75.5 \\
$\left\langle A_{\text {remnant }}\right\rangle$ & 52.7 & 53.4 & 53.5 & 53.0 \\
$\left\langle E^{*} / A_{\text {remnant }}\right\rangle(\mathrm{MeV})$ & 1.82 & 1.30 & 1.65 & 1.49 \\
\hline \hline
\end{tabular}

a selected number of isotopes were measured. For the model curves in Figs. 2 and 3, we have chosen to define the residueproduction cross sections as the sums of the calculated isotopic residue-production cross sections over the nuclides observed in the FRS experiments. No kinematical cut was applied. Since the SPALADIN and FRS data sets are largely compatible, as it clearly appears from Fig. 2, we do not expect this choice to bias our analysis.

For a given deexcitation stage, the curves reflect the differences in the cascade output. Note that calculated cross sections for residues close to the target (say $Z \geqslant 20$ for ${ }^{56} \mathrm{Fe}$ and $Z \geqslant 45$ for ${ }^{136} \mathrm{Xe}$ ) are almost independent of the choice of the deexcitation model and are dominated by the cascade model; in particular, they are consistently better reproduced by INCL4.5 than ISABEL. We consider that an accurate prediction of these cross sections is a crucial prerequisite that the cascade model must satisfy if it is to be used for the study of more exclusive and discriminating observables. Thus, in Sec. V and following, we will retain only the INCL 4.5 model for the analysis of fragment correlations and velocity distributions.

INCL4.5's hotter and lighter remnants also lead, on average, to lighter residues. Note that the IMF cross sections in $p+{ }^{56} \mathrm{Fe}$ and the $10 \lesssim Z \lesssim 30$ cross-section plateau in $p+{ }^{136} \mathrm{Xe}$ are better reproduced by the INCL4.5/deexcitation combinations, while ISABEL consistently underestimates the ${ }^{136} \mathrm{Xe}$ cross sections by a factor of about 3 . Comparison with the excitation-energy distributions in Fig. 1 suggests that these cross sections are associated with highly excited remnants.

TABLE II. Same as in Table I but for $1-\mathrm{GeV} p+{ }^{136} \mathrm{Xe}$.

\begin{tabular}{lcccc}
\hline \hline & INCL4.5 & ISABEL & $\begin{array}{c}\text { INCL4.5 } \\
\text { (no clusters) }\end{array}$ & INCL4.2 \\
\hline$\sigma^{\text {reac }}(\mathrm{mb})$ & 1377 & 1332 & 1381 & 1327 \\
$\left\langle E^{*}\right\rangle(\mathrm{MeV})$ & 139.2 & 116.3 & 132.6 & 113.5 \\
$\left\langle A_{\text {remnant }}\right\rangle$ & 131.4 & 132.4 & 132.6 & 131.7 \\
$\left\langle E^{*} / A_{\text {remnant }}\right\rangle(\mathrm{MeV})$ & 1.08 & 0.89 & 1.02 & 0.88 \\
\hline \hline
\end{tabular}



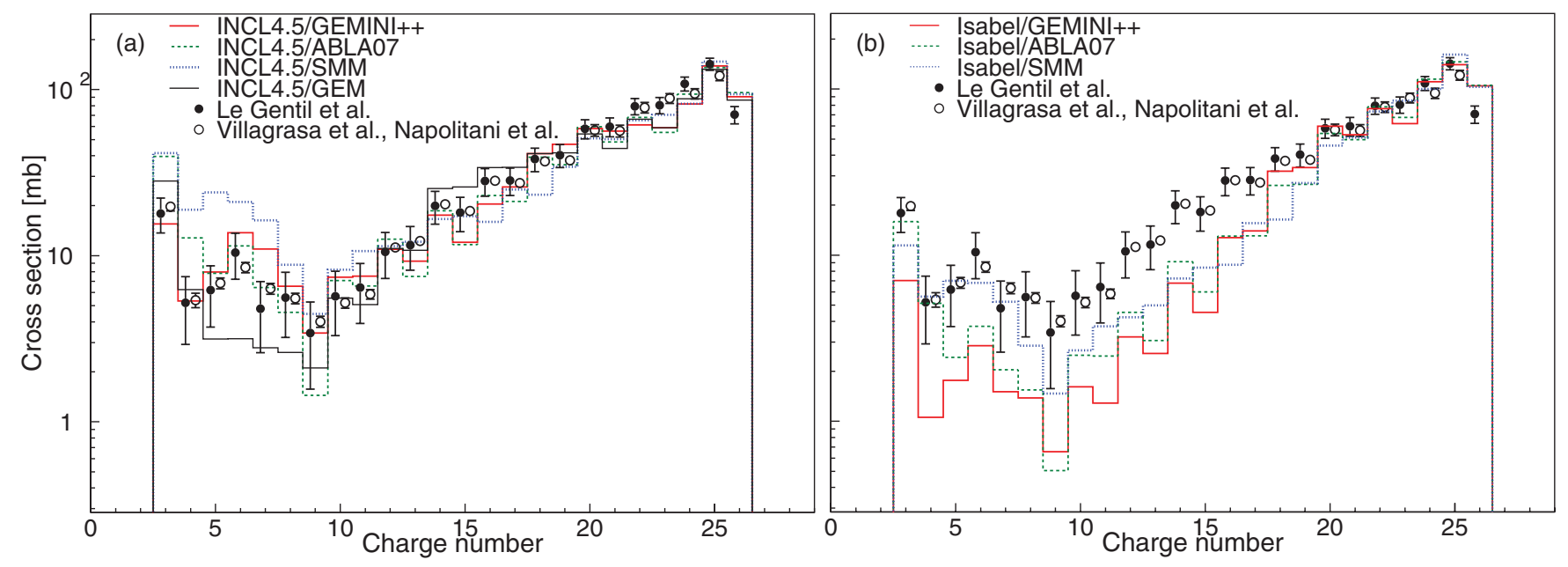

FIG. 2. (Color online) Inclusive residue-production cross sections for $1-\mathrm{GeV} p+{ }^{56} \mathrm{Fe}$, as a function of the nuclide charge. (a) Intranuclear cascade simulated by INCL4.5. (b) ISABEL. Experimental data from Refs. [6,7,9].

Likewise, ISABEL consistently underestimates cross sections with $10 \lesssim Z \lesssim 18$ in $p+{ }^{56} \mathrm{Fe}$.

The sensitivity of the inclusive residue-production cross sections to cascade can be further illustrated by considering the results obtained with the GEM deexcitation model [27], coupled with INCL4.5 (left panels of Figs. 2 and 3). In a previous study [6], the INCL4.2/GEM combination was excluded from the study of SPALADIN correlations because it was unable to reproduce the IMF-production cross sections in $p+{ }^{56} \mathrm{Fe}$. New INCL4.5/GEM calculations predict IMF cross sections that are about a factor of 3 higher than the INCL4.2/GEM and in acceptable agreement with the experimental data; this is due to INCL4.5's different excitation-energy and remnant-mass distributions. However, the plateau cross sections in $p+{ }^{136} \mathrm{Xe}$ are underestimated by at least three orders of magnitude by INCL4.5/GEM. Thus, we also exclude the GEM deexcitation model from this study.

If we now focus on a fixed cascade model (e.g., INCL4.5), we can observe that the three deexcitation models produce similar charge distributions. In this sense, we confirm that residue-production cross sections are rather insensitive to the deexcitation mechanism. However, we remark that deexcitation models present free parameters that can be adjusted to help reproduce the residue-production cross sections. The $p+{ }^{56} \mathrm{Fe}$ data set, in particular, is a popular benchmark for spallation models (cascade/deexcitation) due to its good accuracy. The GEMINI++ parameters connected with asymmetric fission were admittedly fitted to the $p+{ }^{56} \mathrm{Fe}$ and $p+{ }^{136} \mathrm{Xe}$ residue-production cross sections, among other data sets [22]. Thus, Figs. 2 and 3 can deceptively lead to underestimate the sensitivity of residue-production cross sections to the deexcitation model.

The sensitivity to deexcitation can be further appreciated by analyzing how different deexcitation mechanisms contribute to the residue-production cross section.

\section{A. Production mechanism in the deexcitation models}

It is instructive to study how the different deexcitation models reconstruct the residue-production cross sections as the
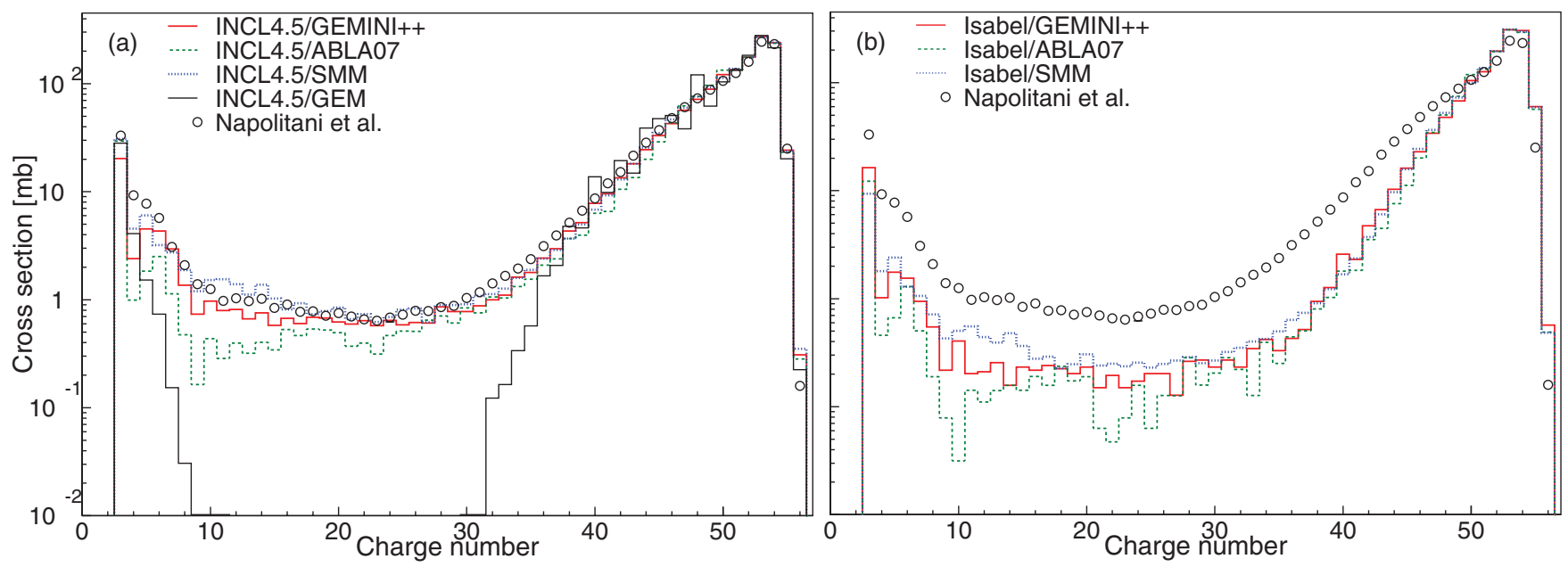

FIG. 3. (Color online) Same as in Fig. 2 but for $1-\mathrm{GeV} p+{ }^{136} \mathrm{Xe}$. Experimental data from Ref. [8]. 


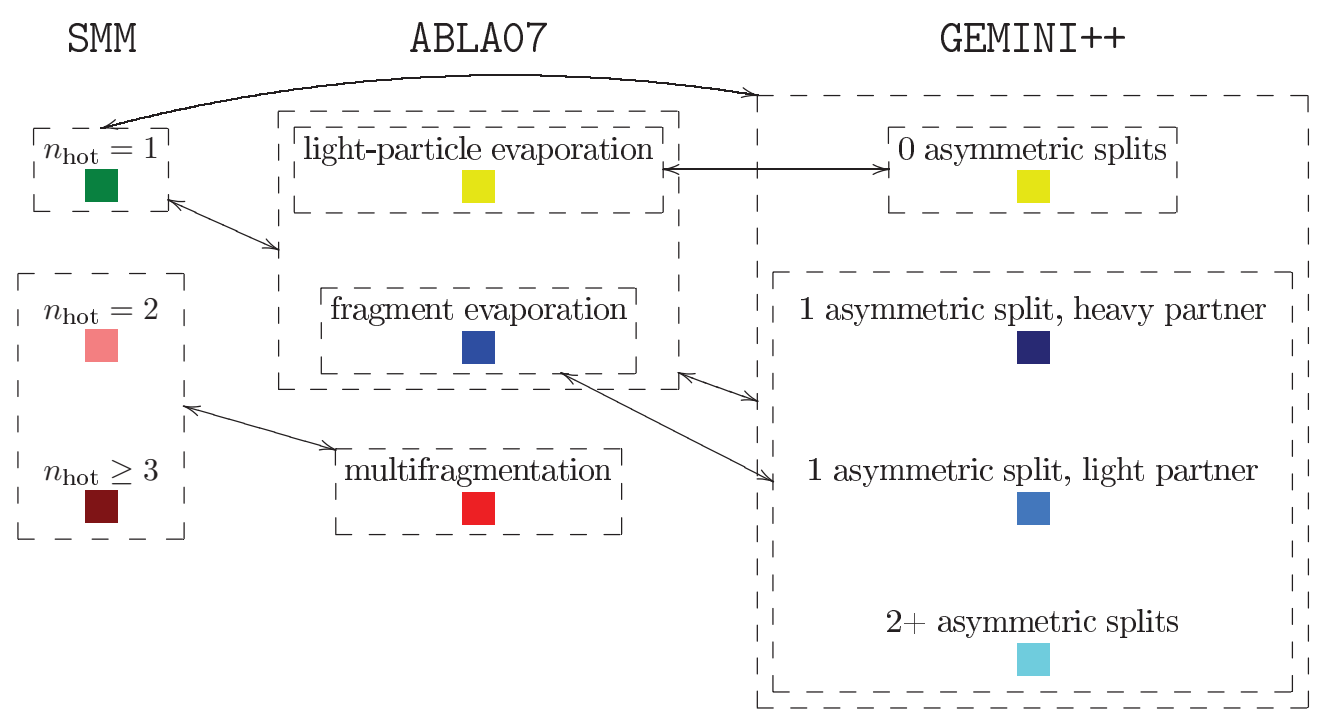

FIG. 4. (Color online) Summary of the partitioning of production mechanisms in the different deexcitation models. Equivalent mechanisms are connected by arrows and are represented by the same or similar colors in Figs. 5-9. See text for more details about the partitioning.

sum of different production mechanisms. However, we need to introduce this discussion by a few important remarks. First, different deexcitation models have different, possibly nonoverlapping, sets of production mechanisms (Sec. II B); thus, each partition must be seen as model dependent and cannot be directly compared to experimental data or to other partitions. Second, although all models internally construct some kind of deexcitation-history tree, only a limited, model-dependent amount of information about the decay history is readily available to the user. Figure 4 summarizes how production mechanisms are partitioned in each model; details about each partitioning will be given in the model-specific discussions that follow. Each deexcitation mechanism is assigned a color, which is consistently used in Figs. 5-9. We attempted to assign similar colors to similar mechanisms. In some cases, a specific mechanism in one model can be considered equivalent to another mechanism or to the sum of other mechanisms in another model. These cases are indicated by arrows and boxes in Fig. 4. The analysis of the production mechanism will focus on the coupling of the deexcitation models with INCL4.5, but the results are qualitatively valid for ISABEL, too.

We start by analyzing SMM (Fig. 5). First, we identify the cascade component of the cross section. The rest of the residue-production cross section is partitioned by labeling each simulated event with the number $n_{\text {hot }}$ of hot fragments that emerge from SMM's initial multifragmentation submodule (and that later deexcite by sequential evaporation).

Figure 5 suggests that the plateau cross sections in $p+{ }^{136} \mathrm{Xe}$ and the IMF cross sections in $p+{ }^{56} \mathrm{Fe}$ are almost entirely due to multifragmentation. However, care must be exercised with this definition of the multifragmentation contribution. First, the onset of multifragmentation in SMM is smooth. Close to the multifragmentation threshold, the most probable breakup configuration is binary, with one breakup partner much larger than the other. Such processes are similar to (and probably indistinguishable from) binary
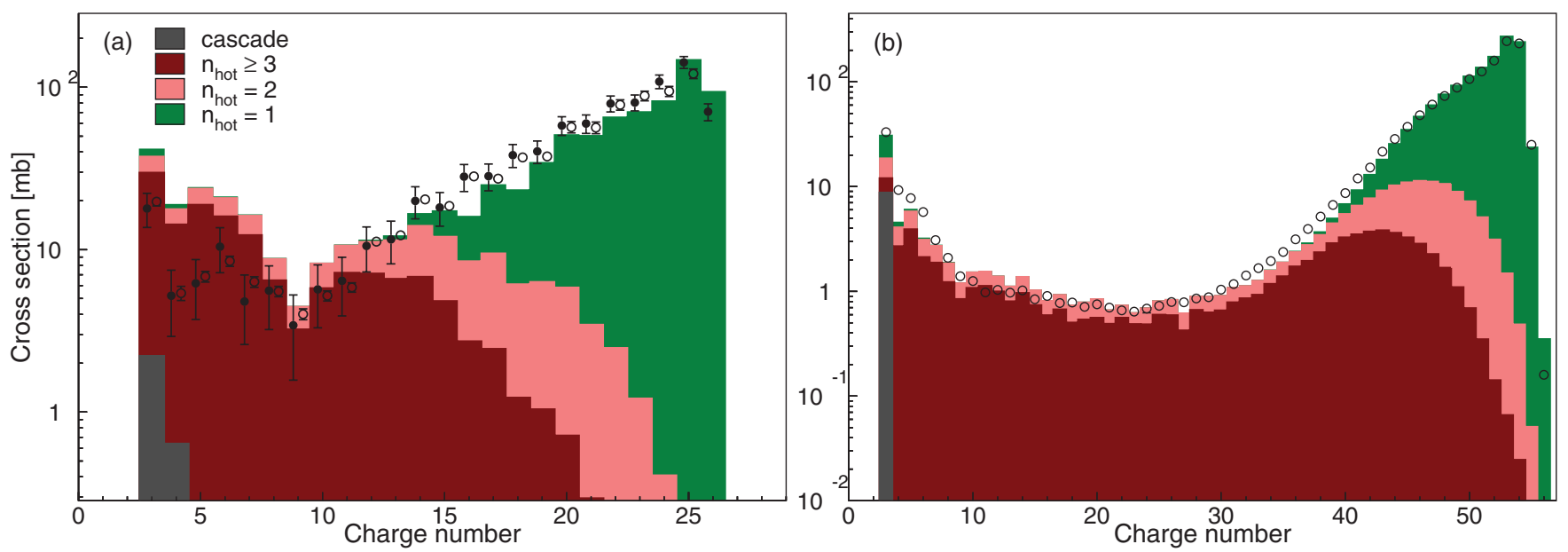

FIG. 5. (Color online) Decomposition of the residue-production cross sections predicted by INCL4.5/SMM according to the number of hot fragments produced in the multifragmentation stage $\left(n_{\text {hot }}\right)$. (a): $p+{ }^{56} \mathrm{Fe}$. (b): $p+{ }^{136} \mathrm{Xe}$. Experimental data from Refs. [6-9]. 

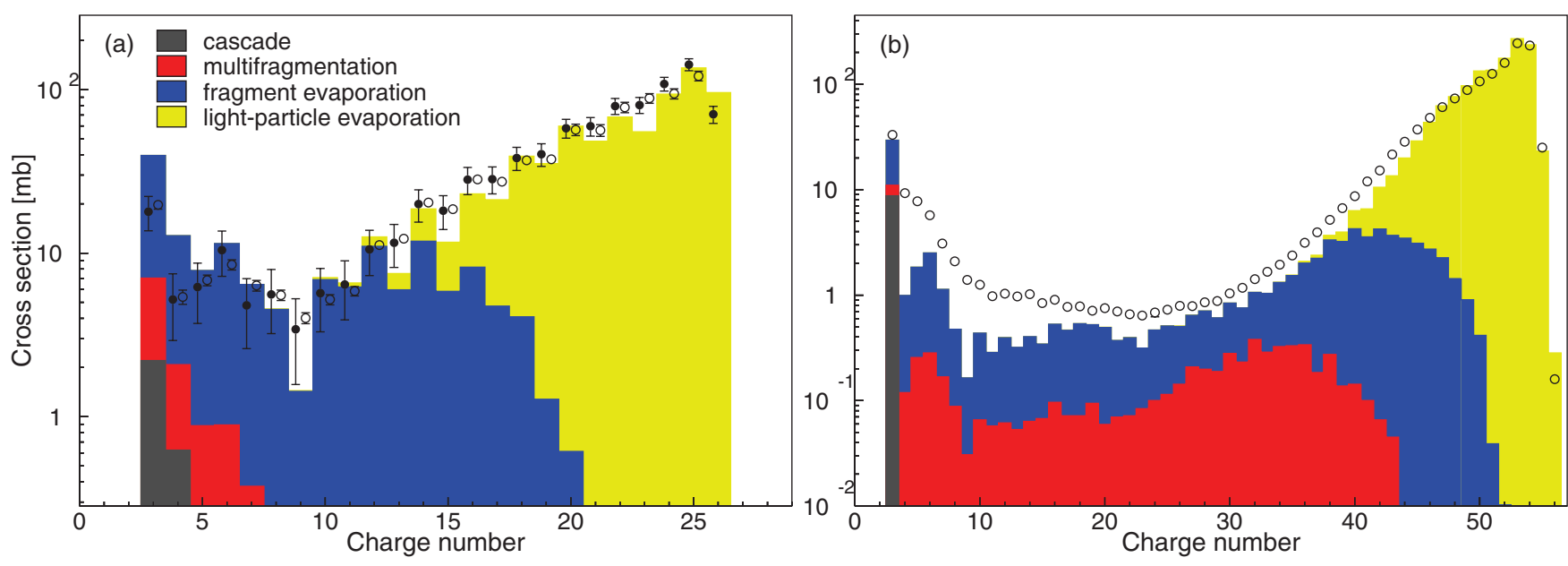

FIG. 6. (Color online) Decomposition of the residue-production cross sections predicted by INCL4.5/ABLA07 according to the deexcitation mechanism. (a) $p+{ }^{56} \mathrm{Fe}$. (b) $p+{ }^{136} \mathrm{Xe}$. Experimental data from Refs. [6-9].

decays and somehow provide a smooth transition to the real multifragmentation regime. Moreover, SMM can produce events where composite fragments are evaporated during the secondary deexcitation of the hot fragments. Whether such events should be counted as multifragmentation is unclear. In our analysis, these events are simply classified according to the multiplicity of hot multifragmentation products. Thus, the importance of multifragmentation for fragment production cannot be easily extracted from the partitioning in Fig. 5 and would require better event labeling, which is unfortunately unavailable at the moment. The predicted cross sections for nominal multifragmentation $\left(n_{\text {hot }} \geqslant 2\right)$, which can be interpreted as upper limits for the "real" multifragmentation cross section, are $146.0 \mathrm{mb}\left({ }^{56} \mathrm{Fe}\right)$ and $137.7 \mathrm{mb}\left({ }^{136} \mathrm{Xe}\right)$, which correspond to $18.7 \%$ and $10.0 \%$ of the respective reaction cross sections.

Figure 6 displays the partitioning of the INCL4.5/ABLA07 cross sections. Events that triggered ABLA07's multifragmentation module are classified as "multifragmentation"; as in the case of SMM, this might include some contamination from secondary fragment evaporation after nominal multifragmentation. Events that did not trigger multifragmentation are catalogued as "fragment evaporation" if one or more fragments were emitted, and as "light-particle evaporation" otherwise. Note that events with excitation energies below the particle-emission threshold are also classified as "light-particle evaporation."

One can observe that the cross sections for nominal multifragmentation are much smaller than in the case of SMM: $13.3 \mathrm{mb}\left(1.7 \%\right.$ of the reaction cross section) for $p+{ }^{56} \mathrm{Fe}$ and $4.6 \mathrm{mb}(0.3 \%)$ for $p+{ }^{136} \mathrm{Xe}$. This is due to ABLA07's higher multifragmentation threshold. According to Eq. (1), the freeze-out temperatures for ${ }^{56} \mathrm{Fe}$ and ${ }^{136} \mathrm{Xe}$ are 7.97 and $6.36 \mathrm{MeV}$, respectively, which correspond (assuming Ignatyuk's level-density parametrization [28]) to excitation energies of 6.22 and 3.70 A MeV. This should be compared with the typical SMM threshold of $3 \mathrm{~A} \mathrm{MeV}$. Since the remnant cross section drops fast as the excitation energy increases, even
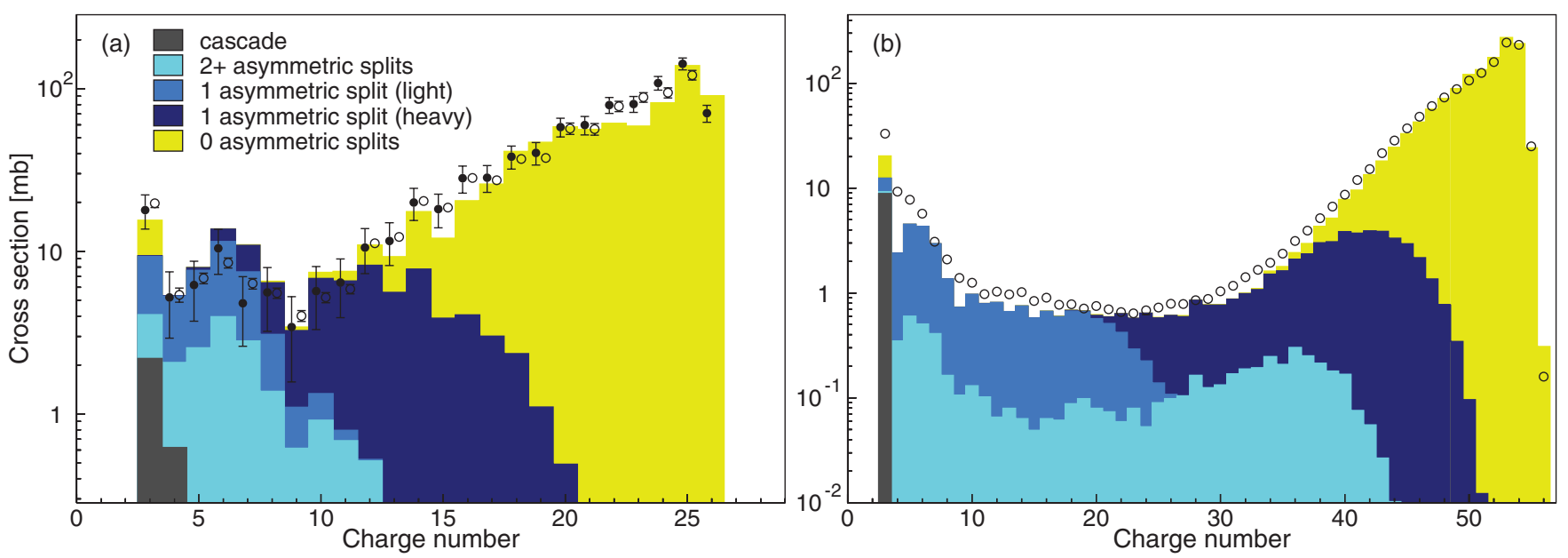

FIG. 7. (Color online) Decomposition of the residue-production cross section predicted by INCL4.5/GEMINI++ according to the number of asymmetric splits leading to a given fragment. (a) $p+{ }^{56} \mathrm{Fe}$. (b) $p+{ }^{136} \mathrm{Xe}$. Experimental data from Refs. [6-9]. 


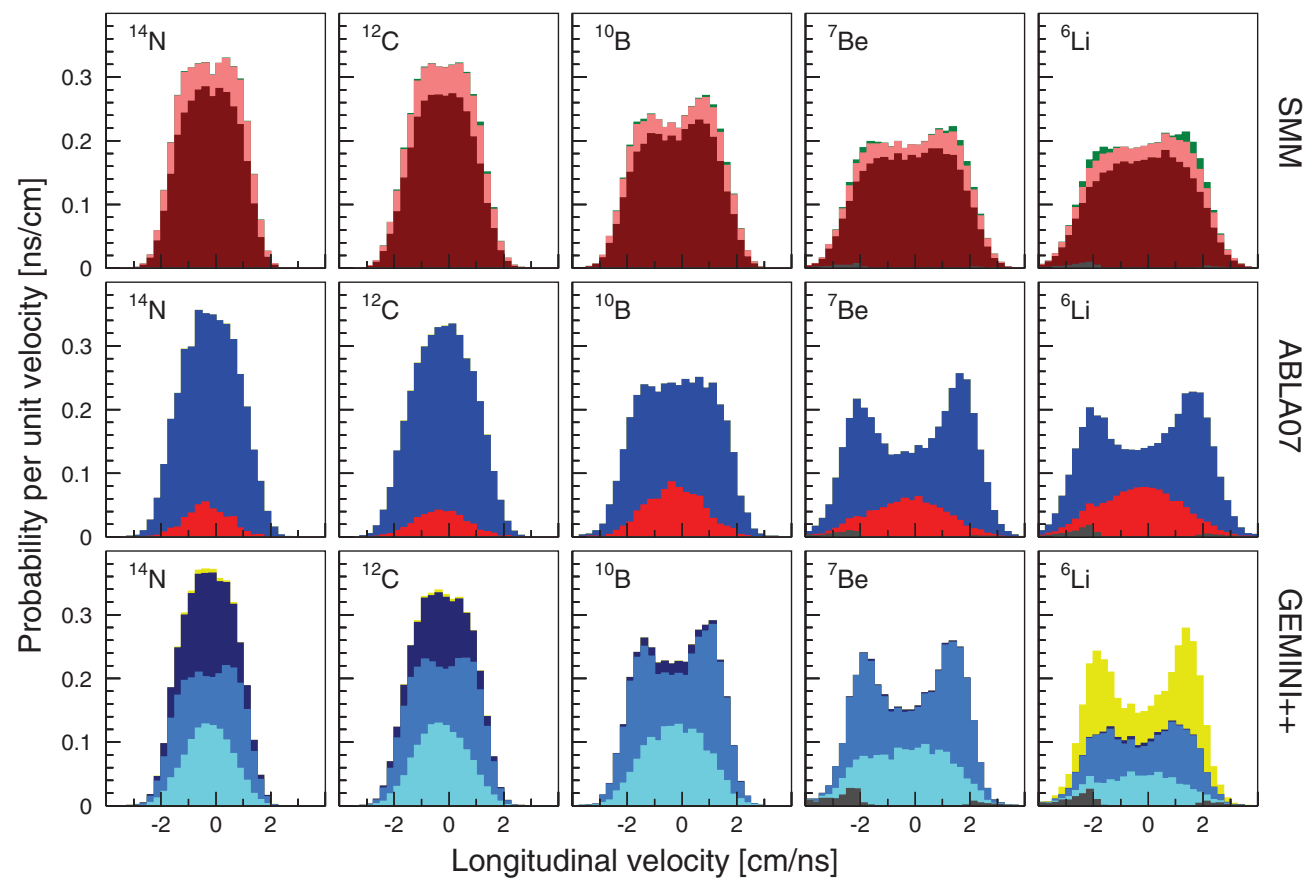

FIG. 8. (Color online) Decomposition of simulated longitudinal-velocity distributions in ${ }^{56} \mathrm{Fe}+{ }^{1} \mathrm{H}$ for some selected nuclides, according to the partitions summarized in Fig. 4.

a moderate difference in the multifragmentation threshold can result in a large cross section difference.

In any case, we stress that most of ABLA07's IMF cross section in $p+{ }^{56} \mathrm{Fe}$ does not originate from multifragmentation events. This is at variance with previous claims [6], based on INCL4.2/GEM, that the $p+{ }^{56}$ Fe residue-production cross sections could not be explained by evaporation. We stress that, first, INCL4.5/GEM provides results similar to INCL4.5/ABLA07 on $p+{ }^{56}$ Feand only fails to describe the $p+{ }^{136} \mathrm{Xe}$ data; second, INCL4.5/ABLA07 is also able to describe the $p+{ }^{136} \mathrm{Xe}$ plateau cross sections mostly thanks to evaporation alone. Therefore, it is not possible to exclude evaporation solely on the basis of the results of one code (GEM) for one system $\left({ }^{56} \mathrm{Fe}\right)$. The details of the evaporation model are obviously important, since ABLA07 is able to provide adequate agreement with all the experimental data considered so far.

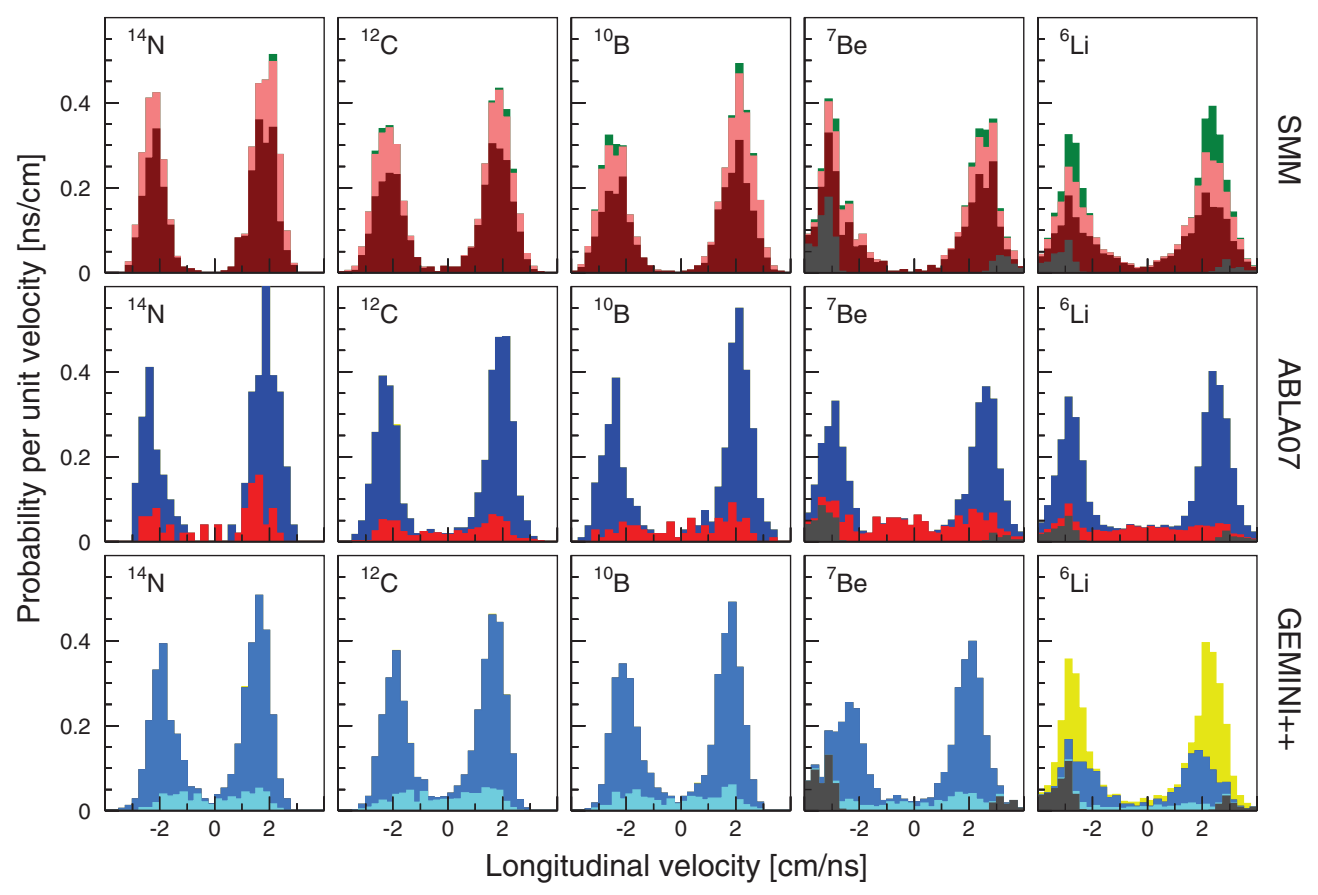

FIG. 9. (Color online) Same as Fig. 8 but for ${ }^{136} \mathrm{Xe}+{ }^{1} \mathrm{H}$. 

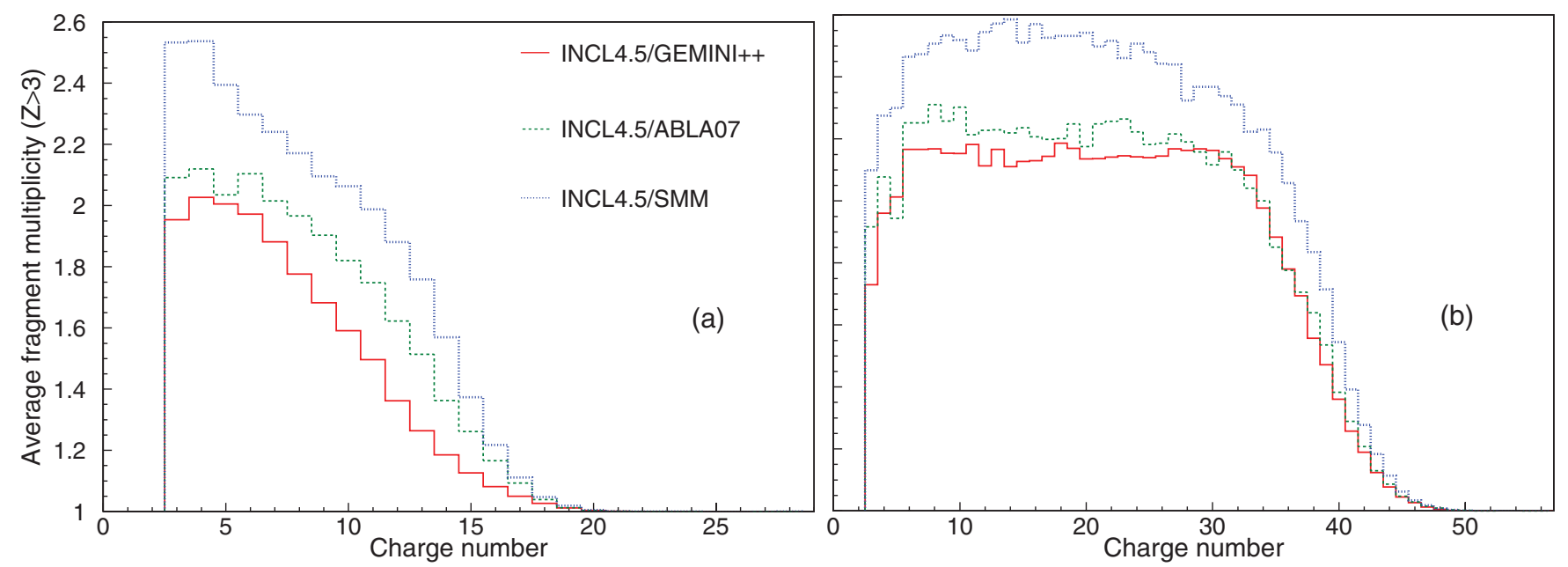

FIG. 10. (Color online) Calculated average multiplicity of particles with $Z \geqslant 3$, as a function of the charge of the fragments appearing in the event. See text for more details. (a) $p+{ }^{56} \mathrm{Fe}$. (b) $p+{ }^{136} \mathrm{Xe}$.

Finally, the INCL4.5/GEMINI++ partitioning is shown in Fig. 7. Deexcitation particles are classified according to the number of asymmetric splits that led to their production. For particles following from only one asymmetric split, we distinguish if they originated from the light or the heavy split partner. For example, if a remnant splits into fragments $A$ and $B$, with $A$ larger than $B$, and $A$ subsequently splits into $C$ and $D, B$ will be tallied in the "1 asymmetric split (light)" histogram, while $C$ and $D$ will be counted as " $2+$ asymmetric splits." Light-particle evaporation $(Z \leqslant 3)$ does not influence the count of asymmetric splits.

Note that we assigned the same color to the GEMINI++ "0 asymmetric splits" and the ABLA07 "light-particle evaporation" components, suggesting that the two mechanisms are equivalent (see also Fig. 4). However, emission of $\mathrm{Li}$ isotopes in GEMINI++ is described by the Hauser-Feshbach evaporation formalism (Sec. II B2) and, therefore, is not counted as an asymmetric split. In ABLA07, on the other hand, emission of $\mathrm{Li}$ fragments is counted as "fragment evaporation." Therefore, the two classes should be considered equivalent for emission of fragments with $Z \geqslant 4$.

The INCL4.5/GEMINI++ calculations predict a small symmetric-fission component in $p+{ }^{136} \mathrm{Xe}$. Although ${ }^{136} \mathrm{Xe}$ is below the Businaro-Gallone point, a small number of symmetric-fission event do occur in remnants with high values of spin and of the $Z^{2} / A$ ratio. The cross section for such events is only about $5 \times 10^{-5}$ times the reaction cross section and it is not visible on the scale of Fig. 7.

Perhaps unsurprisingly, we find that GEMINI++ generates most of the IMF cross section in $p+{ }^{56} \mathrm{Fe}$ and all the plateau cross section in $p+{ }^{136} \mathrm{Xe}$ through the asymmetricsplitting mechanism. For $Z=3$ the contribution with no asymmetric split corresponds to evaporated $\mathrm{Li}$ nuclei (we remind that emission of nuclei up to $Z=3$ is described by the evaporation formalism; see Sec. II B2). There is a striking similarity between the right panels of Figs. 6 and 7. The contributions of nominal multifragmentation (for ABLA07) and of pseudomultifragmentation 2+-split events (for GEMINI++) in $p+{ }^{136} \mathrm{Xe}$ are approximately of the same magnitude and show a similar $Z$ dependence. This suggests that ABLA07 and GEMINI++ also predict similar fragment multiplicities in $p+{ }^{136} \mathrm{Xe}$, which is indeed shown to be the case in Fig. 10. The curves represent the average number of particles with $Z \geqslant 3$ as a function of the charge of the fragments that appear in the event. Thus, for example, INCL4.5/GEMINI ++ predicts that neon fragments $(Z=10)$ from $p+{ }^{136} \mathrm{Xe}$ appear in events with on average $\sim 2.1$ particles with $Z \geqslant 3$ (including the $\mathrm{Ne}$ fragment itself). Interestingly, INCL4.5/GEMINI++ and INCL4.5/SMM predict quite different average fragment multiplicities in the ${ }^{136} \mathrm{Xe}$ plateau region, despite the residueproduction cross sections being very similar. In general, the average multiplicity does not seem to strictly correlate with the residue-production cross section. This finding manifestly calls for more exclusive observables, such as multiplicity distributions and fragment correlations. Such data do exist for $p+{ }^{56} \mathrm{Fe}$ (see the following section); the analysis of a SPALADIN-type $p+{ }^{136} \mathrm{Xe}$ experiment has recently been completed and will soon be published [29].

As a final remark, we underline that light-charged particles emitted during the cascade stage might play some role in the determination of cross section for the lowest values of $Z$. By default, INCL4.5 only produces clusters with $A \leqslant 8, Z \leqslant 5$. The cascade contribution drops off at $Z=4$ because none of the selected $Z=5$ isotopes has $A \leqslant 8$ (see the beginning of Sec. IV for the discussion on the FRS isotope selection). Almost $50 \%$ of the $Z=3$ cross section in $p+{ }^{136} \mathrm{Xe}$ comes from cascade ${ }^{6,7} \mathrm{Li}$. It is not clear whether heavier clusters might significantly contribute to the $Z \geqslant 4$ cross sections, which seem to be slightly underestimated by the models.

\section{SPALADIN CORRELATIONS}

We now turn to the analysis of the model predictions for the SPALADIN $p+{ }^{56} \mathrm{Fe}$ data set [6]. Adequate reproduction of inclusive observables is a prerequisite for the study of semiexclusive correlations and/or multiplicity distributions. 
Therefore, in what follows we will only retain the INCL4.5 cascade model, which gives residue-production cross sections that are in better agreement with the experimental data (see Sec. IV).

The goal of the SPALADIN experiment was to measure observables in coincidence for the $1-\mathrm{GeV} p+{ }^{56} \mathrm{Fe}$ reaction in inverse kinematics. These semiexclusive measurements were obtained at the price of a more sophisticated setup and experimental analysis than typical inclusive experiments. In Ref. [6] events generated by cascade/deexcitation models were filtered through a GEANT4 transport calculation and analyzed like the experimental data, providing little evidence of convincing multifragmentation signatures.

Since cascade and deexcitation models are in continuous evolution, it is necessary to periodically verify their predictions on sensitive semiexclusive observables. The INCL4.5 and GEMINI++ codes, for example, have largely evolved since the publication of Ref. [6]. Moreover, the old version of the ABLA code did not allow emission of any nucleus heavier than $\alpha$ particles, was, thus, unsuccessful at reproducing inclusive residue-production cross sections, and was, therefore, excluded from the study of correlations in Ref. [6]. The new ABLA07 code, as proven above, can adequately describe the residue-production cross sections; it is, therefore, interesting to test its predictions for the SPALADIN correlations. Such considerations provide the motivation for this section.

We start by analyzing the decomposition of the residueproduction cross sections. Experimental events were subdivided into five classes, according to the number of fragments $(Z \geqslant 3)$ and helium nuclei that were detected. Modelled events were run through a GEANT4 filter that reproduces the experimental setup and simulates detector efficiency and, subsequently, categorized just like the experimental events. Figure 11 displays the result of this exercise. Clearly, INCL4.5/GEMINI++ and INCL4.5/ABLA07 yield the best description of the experimental data. The appraisal of SMM's results in the IMF region appears to be biased by its slight overestimation of the residue-production cross sections. Note also that SMM's and ABLA07's overestimation of 3+-fragment events, which are properly reproduced by GEMINI++, follow the same trend as the average fragment multiplicities depicted in Fig. 10. The INCL4.5/SMM and INCL4.5/GEMINI++ results are qualitatively similar to those presented in Ref. [6], although both the cascade model and the deexcitation models differ sensibly.

We now address correlations between fragment charges. We restrict our attention to events with at least two detected fragments $(Z \geqslant 3)$ and we define $Z_{1}$ and $Z_{2}$ to be the largest and the second-largest observed charges. For these events, we define three bins in detected neutron-plus-helium multiplicity (1-2, 3-4, and 5-6), which is expected to be fairly correlated with the excitation energy of the cascade remnant. The correlation between detected neutron-plus-helium multiplicity and excitation energy was studied with INCL4.2 and was shown to be essentially independent of the deexcitation model [30]; however, our results contradict this conclusion. Table III shows the average excitation energies per nucleon in events with two $(Z \geqslant 3)$ fragments as a function of the neutron-plus-helium multiplicity. For comparison, we also provide the values
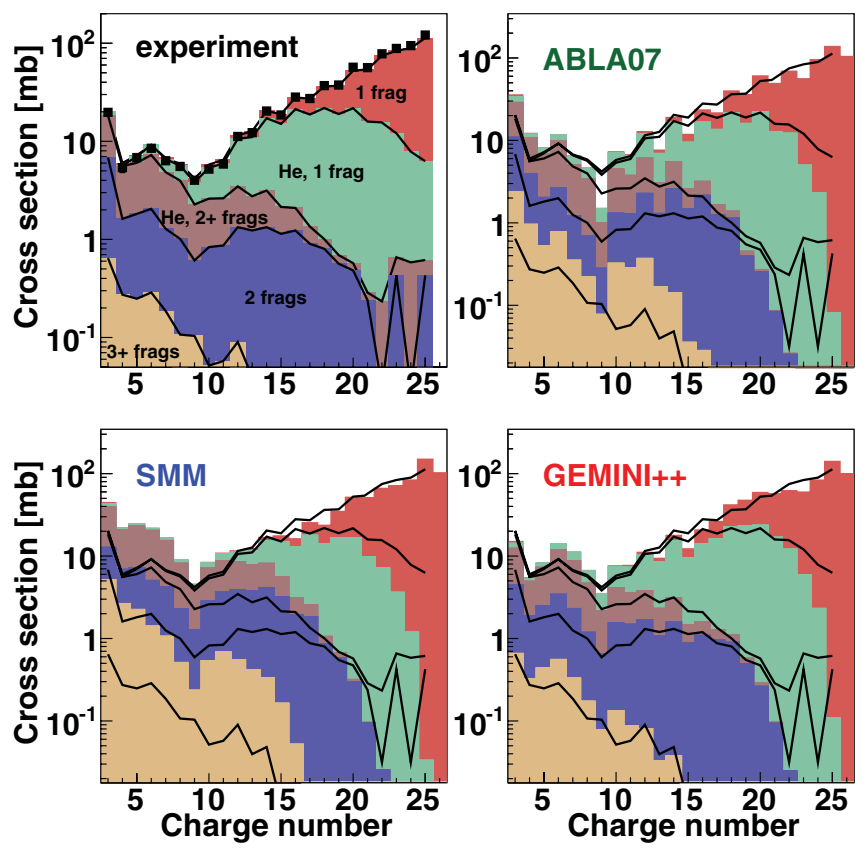

FIG. 11. (Color online) Partitioning of the $1-\mathrm{GeV} p+{ }^{56} \mathrm{Fe}$ cross section according to the number of observed helium nuclei and IMFs. Solid lines represent the experimental data and histograms represent the model predictions. The cascade model is INCL4.5. Experimental data from Ref. [6].

computed by Le Gentil et al. with INCL4.2 [6], which were claimed to be independent of the deexcitation model [30]. First, we observe that the average excitation energies are not independent of the deexcitation model, at least for the high-multiplicity bin. Note, however, that the distributions of excitation energies within each bin are broader than the differences in average excitation energies among neighboring bins; thus, the detected neutron-plus-helium multiplicity cannot be interpreted as a precise measure of the remnant excitation energy. Second, the average excitation energies that we find with INCL4.5 are consistently higher than those determined by Le Gentil et al. using INCL4.2. Thus, the correlation between neutron-plus-helium multiplicity and excitation energy depends at least on the cascade model; neutron-plus-helium multiplicities cannot represent a universal, model-independent measure of the remnant excitation energy.

Figure 12 shows the distributions of the $Z_{1}-Z_{2}$ difference in the three multiplicity bins. As explained in Ref. [6], the experimental data indicate that asymmetric $\left(Z_{1} \gg Z_{2}\right)$ and symmetric $\left(Z_{1} \simeq Z_{2}\right)$ charge configurations are favored at low and high excitation energy, respectively. All the models reflect this qualitative trend, although only GEMINI++ and ABLA07 are able to reproduce the absolute cross sections with good accuracy. SMM's overestimation is qualitatively consistent with its predictions of IMF yields; in fact, we observe that the cross sections calculated with a given model, if summed over $Z_{1}-Z_{2}$ and over the multiplicity bins, are quantitatively comparable to the IMF-production cross sections shown in Fig. 2.

Compared to Le Gentil et al.'s results, our work confirms that INCL4.5/GEMINI++ provides the best description of the 
TABLE III. Remnant excitation energies per nucleon in $1-\mathrm{GeV} p+{ }^{56} \mathrm{Fe}$ in events with two detected fragments with $Z \geqslant 3$, for three bins in detected neutron-plus-helium multiplicity, as calculated by the three deexcitation models used in this work (coupled to INCL4.5) and by the INCL 4.2 calculations performed by Le Gentil et al. [6]. The values should be interpreted as mean value \pm root mean square.

\begin{tabular}{lccc}
\hline \hline Model & & $E^{*} / A_{\text {remnant }}(\mathrm{MeV})$ & \\
\cline { 2 - 4 } & $1 \leqslant M_{n}+M_{\mathrm{He}}<3$ & $3 \leqslant M_{n}+M_{\mathrm{He}}<5$ & $5 \leqslant M_{n}+M_{\mathrm{He}}<7$ \\
\hline INCL4.5/SMM & $4.4,1.3$ & $4.8,1.4$ & $5.3,1.4$ \\
INCL4.5/ABLA07 & $4.4,1.1$ & $4.7,1.1$ & $5.2,1.1$ \\
INCL4.5/GEMINI++ & $4.2,1.5$ & $4.9,1.5$ & $5.6,1.5$ \\
INCL4.2 & 3.1 & 3.8 & 4.5 \\
\hline \hline
\end{tabular}

$\overline{\bar{a} \text { Considered independent of the deexcitation model in Ref [6]. Root-mean-square values were not provided. See text and Refs. [6,30] }}$ for more details.

$Z_{1}-Z_{2}$ distributions. The new ABLA07 model, when coupled with INCL4.5, also provides a very good reproduction of the experimental data. However, one should also remark that the shapes of the INCL4.5/SMM distributions are now quite similar to the experimental data, which was not the case in Ref. [6], and only the normalization seems to be consistently off by a factor of about 2 . This is quantitatively consistent with INCL4.5/SMM's overestimation of the residue-production cross sections (Fig. 2) and raises an interesting question, i.e., whether it might be possible to adjust INCL4.5/SMM to better reproduce residue-production cross sections and the SPALADIN observables at the same time.

We finally turn to fragment multiplicities. We first need to define the $Z_{\text {bound }}$ variable as the sum of all the detected charges with $Z \geqslant 2$. This quantity was previously found to be negatively correlated with the excitation energy of the cascade remnant, and the correlation was found to be independent of the deexcitation model [6]. Figure 13 demonstrates that both these properties stay true when the calculations are performed with INCL4.5. The $E^{*}-Z_{\text {bound }}$ correlation, however, is found to differ slightly for INCL4.2 and INCL4.5. This indicates that $Z_{\text {bound }}$ should not be considered as a universal, cascade-model-independent measure of the excitation energy, although it seems relatively robust with respect to the choice of the deexcitation model.
The average fragment multiplicities (i.e., the average number of fragments with a given charge produced in a reaction) are plotted in Fig. 14, for fragment charges between 2 and 7, as functions of $Z_{\text {bound }}$. All multiplicities rise as $Z_{\text {bound }}$ decreases, reflecting the positive correlation between fragment multiplicity and excitation energy. Note that production cross sections for $Z=2$ were measured by the SPALADIN collaboration, but they were not plotted on Fig. 2 because we summed the calculated isotopic cross sections over the isotopes measured in the FRS experiments (see Sec. IV).

The first striking result is that, for a fixed fragment, all the curves have a very similar shape. If $Z_{\text {bound }}$ is interpreted as the excitation energy of the cascade remnant, this indicates that all the deexcitation models predict a similar dependence of the fragment-emission probability on the excitation energy. The overall level of the curve is charge-by-charge correlated with the IMF yields in Fig. 2. Thus, for example, ABLA07 and SMM predict too-large multiplicities and too-large production cross sections for $Z=3$. In addition to this correlation, it is not clear whether there is a lesson to be learned from these observables. The strong even-odd staggering at low $Z_{\text {bound }}$ is reproduced by all models.

Summarizing, the quest for model-independent measures of the remnant excitation energy is still open. We checked that
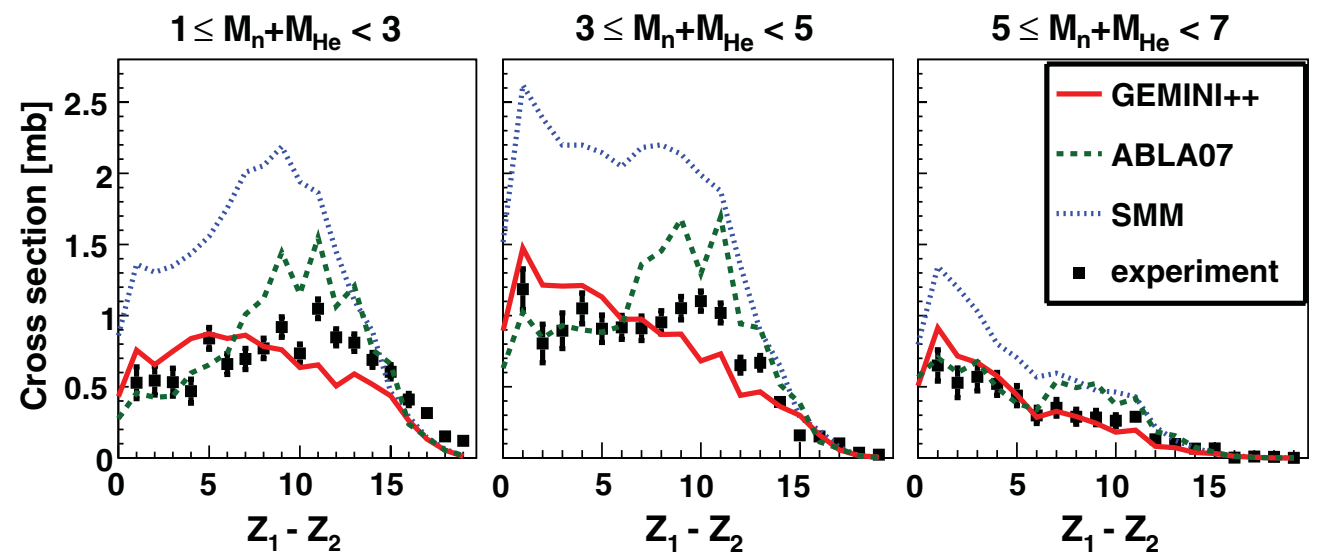

FIG. 12. (Color online) Distributions of $Z_{1}-Z_{2}$ (see text) for three different bins in neutron-plus-helium multiplicity, in events with at least two fragments with $Z \geqslant 3$. See the text for more details about the excitation energies sampled in these bins. The cascade model is INCL4.5. Experimental data from Ref. [6]. 


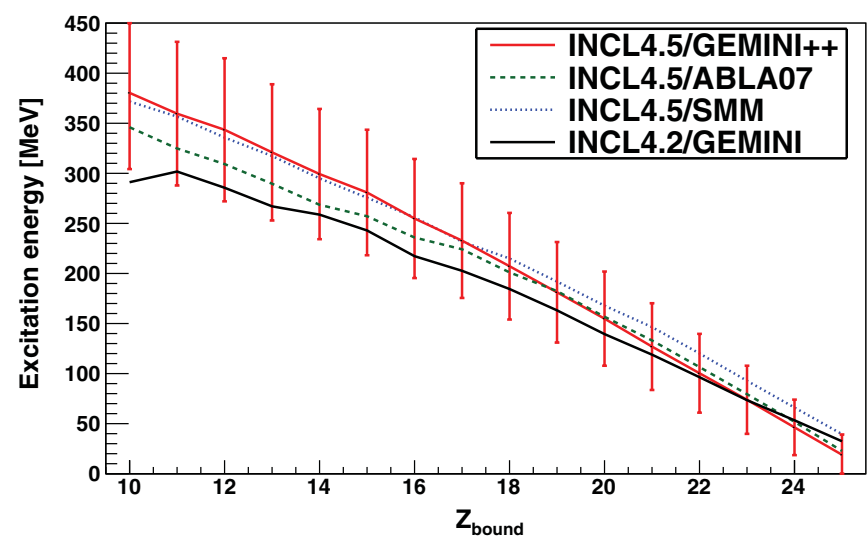

FIG. 13. (Color online) Average remnant excitation energy as a function of the value of $Z_{\text {bound }}$, which is defined as the sum of all the detected charges greater than 1 . The error bars represent the width of the excitation-energy distributions for INCL4.5/GEMINI++. The black line was extracted from Ref. [6] and was computed with INCL4.2/GEMINI. neutron-plus-helium multiplicities and the $Z_{\text {bound }}$ variable are at least sensitive to the cascade model. The new GEMINI ++ and SMM versions yield predictions similar to those reported by Ref. [6], although the cascade model used in the present work yields rather different excitation-energy and remnantmass distributions. The ABLA07 and GEMINI++ models can reproduce most of the considered observables. Given the very small fraction of nominal multifragmentation events predicted by INCL4.5/ABLA07 (Sec. IV A), we confirm that explanation of the SPALADIN data does not require any strong multifragmentation component.

To our knowledge, no published correlation data exist for the $p+{ }^{136} \mathrm{Xe}$ reaction around $1 \mathrm{GeV}$. However, a SPALADINtype experiment was performed in April 2009 and the results of the analysis are due to be published soon [29].

\section{LONGITUDINAL-VELOCITY DISTRIBUTIONS}

Insight about the deexcitation mechanism can also be gained by examining the kinematics of the decay products.
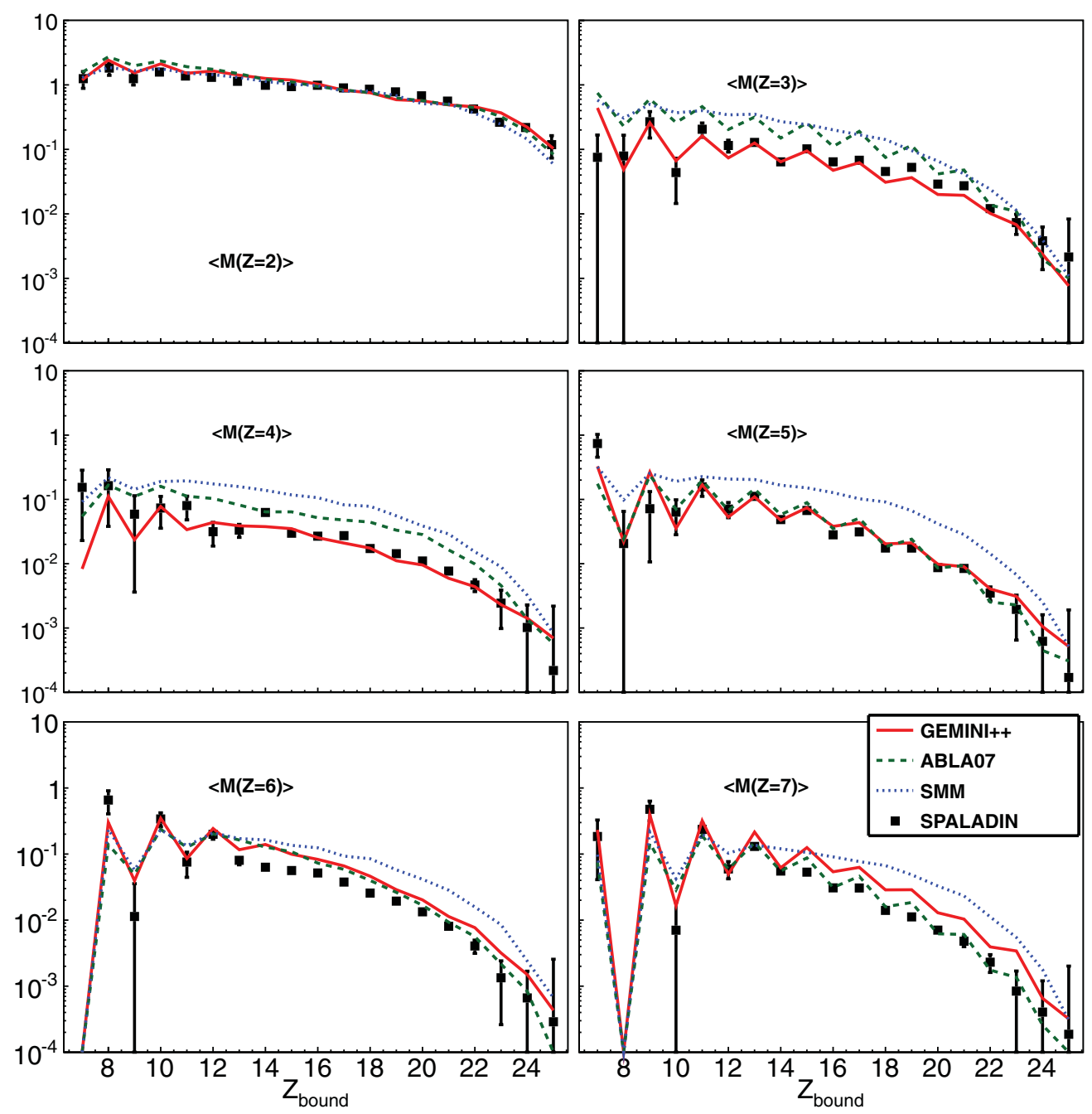

FIG. 14. (Color online) Average fragment multiplicities as a function of the $Z_{\text {bound }}$ variable (see text). The cascade model is INCL4.5. Experimental data from Ref. [6]. 
Sequential binary splits should produce kinematical patterns reminiscent of the decay barriers; multifragmentation, on the other hand, is expected to produce fragments with broad, structureless velocity distributions. We discuss here the velocity distributions measured in the context of the FRS experiments considered in Sec. IV $[9,10]$. The emission velocities of fragments from the $1-\mathrm{GeV} p+{ }^{56} \mathrm{Fe}$ and $p+{ }^{136} \mathrm{Xe}$ reactions are measured using forward spectrometry techniques. Reactions are studied in inverse kinematics, i.e., as a $1 A \mathrm{GeV}^{56} \mathrm{Fe}$ or ${ }^{136} \mathrm{Xe}$ beam impinging on a ${ }^{1} \mathrm{H}$ target. Most of the deexcitation

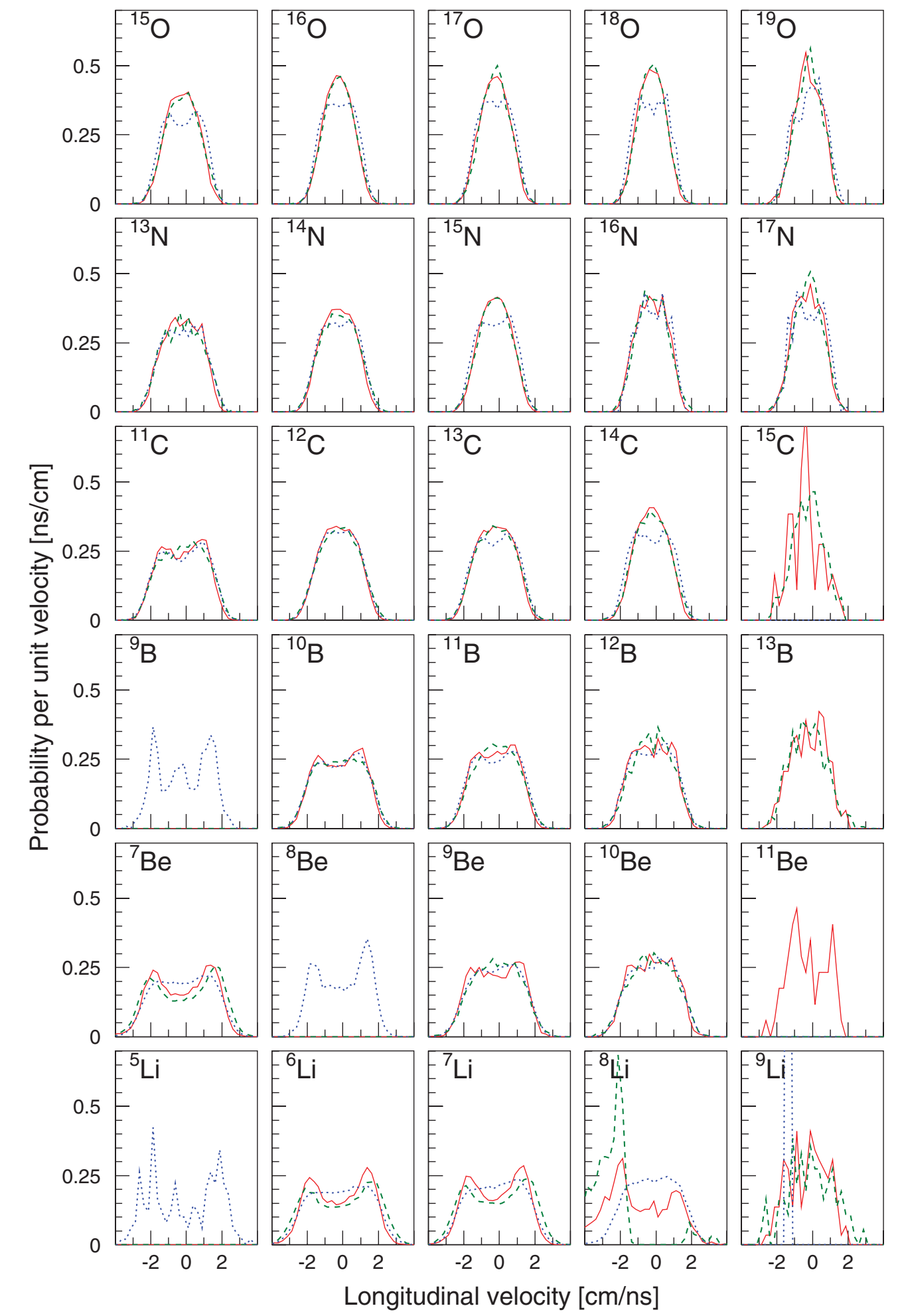

FIG. 15. (Color online) Calculated longitudinal-velocity distributions for $1 A \mathrm{GeV}{ }^{56} \mathrm{Fe}+{ }^{1} \mathrm{H}$ in the beam rest frame. (Red solid line) INCL4.5/GEMINI++, (green dashed line) INCL4.5/ABLA07, (blue dotted line) INCL4.5/SMM. 
products are focalized in a cone around the beam axis. The experimental angular acceptance somewhat depends on the trajectory azimuth, but it is, on average, equal to $15 \mathrm{mrad}$. Particles that satisfy the acceptance cut are identified by mass and charge and their longitudinal velocity (the component of the velocity along the beam axis) is measured. References [9] and [10] report measured longitudinal-velocity distributions (LVDs) for several nuclides with $A \geqslant 6$.

We used our cascade/deexcitation tools to calculate the longitudinal-velocity distributions for the same nuclides. A detailed comparison with the experimental LVDs requires knowledge of the azimuthal dependence of the FRS angular acceptance and a three-dimensional macroscopic transport calculation of the reaction products in the spectrometer. We limited ourselves to a simpler approach: We assumed the angular acceptance to be independent of the trajectory azimuth and equal to the experimental average value of $15 \mathrm{mrad}$. In other words, the acceptance of our simulation is a circular cone in velocity space, centered on the beam axis, with vertex in the origin and aperture of $15 \mathrm{mrad}$. This prevents a refined quantitative comparison of our results with the experimental data, but the emerging trend is nonetheless clear, as we will show in the following.

Figure 15 shows the calculated LVDs for ${ }^{56} \mathrm{Fe}+{ }^{1} \mathrm{H}$. Each distribution is separately normalized to 1 . Note that, in this and all the following figures, the longitudinal velocities refer to the rest frame of the ${ }^{56} \mathrm{Fe}$ projectile, with the proton impinging with negative velocity. This choice was made for consistency with the experimental data plotted in Fig. 16.

We note that a few short-lived nuclides are present in the SMM results $\left({ }^{5} \mathrm{Li},{ }^{8} \mathrm{Be}\right.$, and $\left.{ }^{9} \mathrm{~B}\right)$. These nuclides would typically decay before being detected by the experimental apparatus. The decays could, in principle, populate other IMF species and modify their LVDs, but the nuclides above entirely decay in nucleons and $\alpha$ particles. Therefore, we can neglect them in the following discussion.

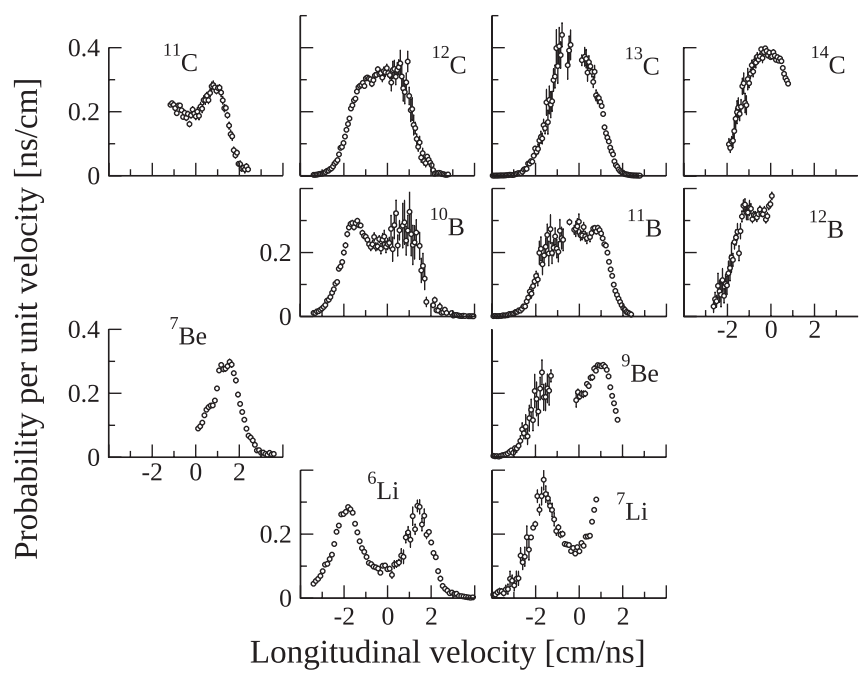

FIG. 16. (Color online) Experimental longitudinal-velocity distributions for $1 A \mathrm{GeV}{ }^{56} \mathrm{Fe}+{ }^{1} \mathrm{H}$ in the beam rest frame. Adapted from Ref. [9].
One observes that all models produce similar, single-peaked distributions for the heaviest IMFs (say for $A \geqslant 9$ ). Only for the lightest IMFs can we observe differences among the model predictions, with GEMINI++ and ABLA07 often producing double-peaked distributions, whereas SMM typically yields flat distributions. These predictions should be compared with the measured distributions (see Fig. 10 in Ref. [9]) which are reported in Fig. 16 for convenience of the reader. In stark contrast with Napolitani et al.'s claims, we find that binary decay does not imply sharp Coulomb holes in the velocity distributions. Indeed, the shapes of the measurements distributions seem to be best described by GEMINI++; compare, e.g., the double-peaked structure of the ${ }^{6,7} \mathrm{Li}$ distributions, where the Coulomb peaks predicted by GEMINI++ are possibly even too weak to account for the measured shape.

How can GEMINI++ produce single-peaked LVDs by relying on its binary-decay mechanism? This question can be answered by partitioning LVDs according to the production mechanism, as done in Fig. 8. First, we observe that nuclei that follow from two or more asymmetric splits (cyan component) expectedly produce single-peaked distributions. The " 0 asymmetric splits" component, which is only present in $\mathrm{Li}$ isotopes, corresponds to direct evaporation and shows a sharp Coulomb hole. However, contrary to what one would naively expect, nuclei following from one asymmetric split produce only mildly structured LVDs. Distributions associated with light split partners do show Coulomb holes, although secondary deexcitation (evaporation of light particles) after the split somewhat blurs the peaks. However, and more importantly, the heavy partner of asymmetric splits typically picks up very little recoil but can retain enough excitation energy to lose much of its mass and be eventually detected as an IMF with small longitudinal velocity. Thus, a sizable fraction of the single-peaked contribution to the GEMINI++ LVDs in Fig. 8 comes from the "1 asymmetric split (heavy)" (dark blue) mechanisms, which we may term the the deexcitation-residue component. This result somewhat contrasts with the interpretation suggested by SMM, which entirely attributes the singlepeaked component to nominal multifragmentation events. Finally, observe that the ABLA07 "fragment evaporation" component (blue), which contains both evaporated fragments and fragment-evaporation residues, shows no stark Coulomb structure for $A \geqslant 10$, consistently with the conclusions suggested by GEMINI ++ .

We stress that the participation of deexcitation residues to IMF distributions is possible only because cascade remnants in $p+{ }^{56} \mathrm{Fe}$ are relatively close in $A$ and $Z$ to the IMF mass region (Fig. 1). The residue component somewhat provides a background noise over which true multifragmentation signatures are superimposed. One of the motivations for studying the $p+{ }^{136} \mathrm{Xe}$ reaction is exactly that the residue component is expected to be completely negligible in the IMF region. It is, therefore, of great interest to consider how well GEMINI++ performs at reproducing the measured LVDs for this reaction.

As displayed in Figs. 17 and 18, the LVDs predicted by GEMINI++ show clear Coulomb holes over all the considered mass and charge range. Indeed, the residue component is 


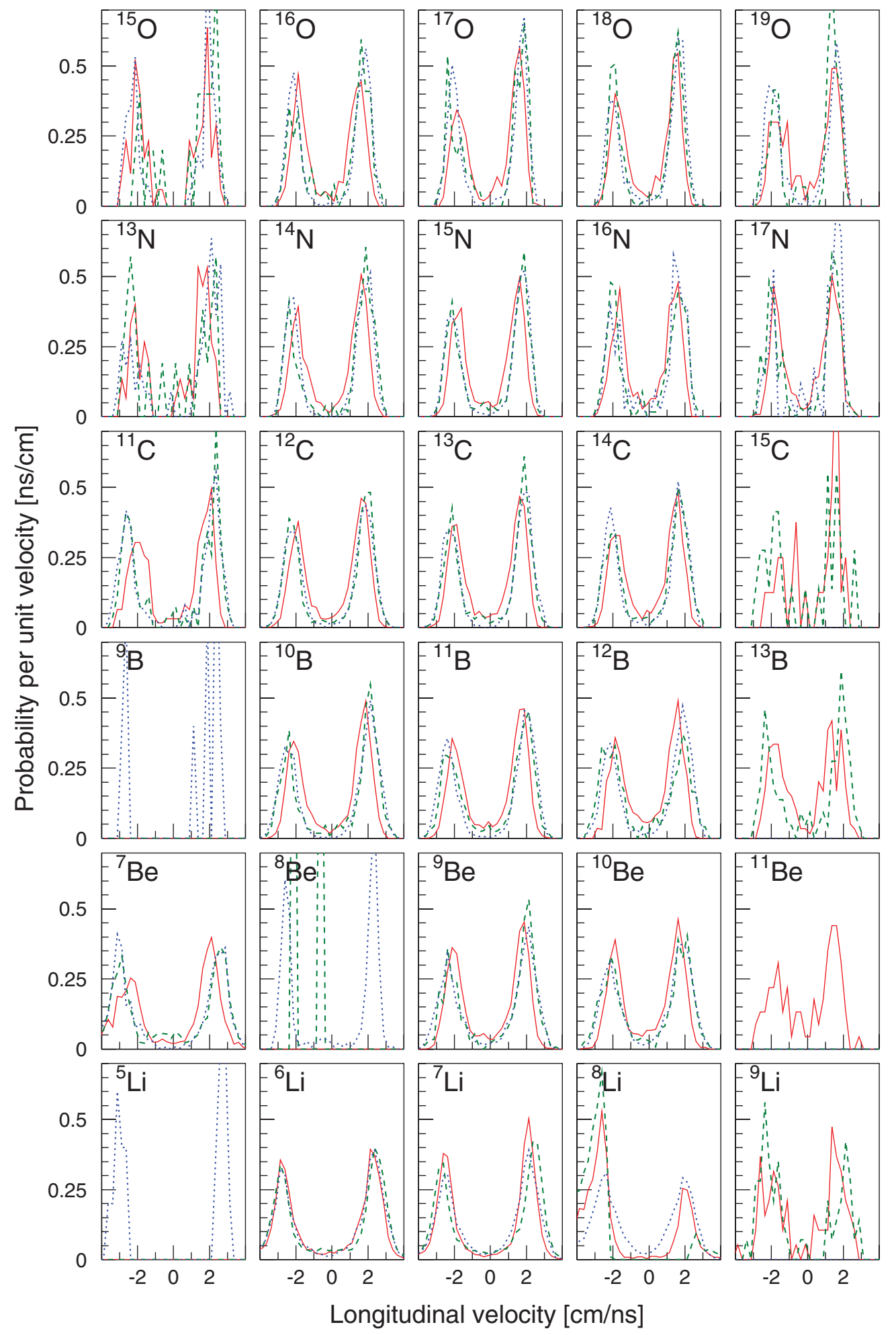

FIG. 17. (Color online) Same as Fig. 15, for $1 A \mathrm{GeV}{ }^{136} \mathrm{Xe}+{ }^{1} \mathrm{H}$.

negligible and GEMINI++ is not able to reproduce the experimental distributions (see Fig. 2 in Ref. [10]), which become rather flat from $Z \simeq 6$, as in the case of ${ }^{56} \mathrm{Fe}$. Surprisingly, however, even ABLA07 and SMM predict doublepeaked LVDs that are very similar to GEMINI++'s, even for relatively heavy nuclides such as the Si isotopes (Fig. 18).
No model seems to be able to account for the single-peaked component that clearly dominates the experimental LVDs for $Z \gtrsim 6$.

This unanticipated result clashes with the widespread belief that multifragmentation should yield single-peaked velocity distributions, especially for high fragment multiplicities. SMM 


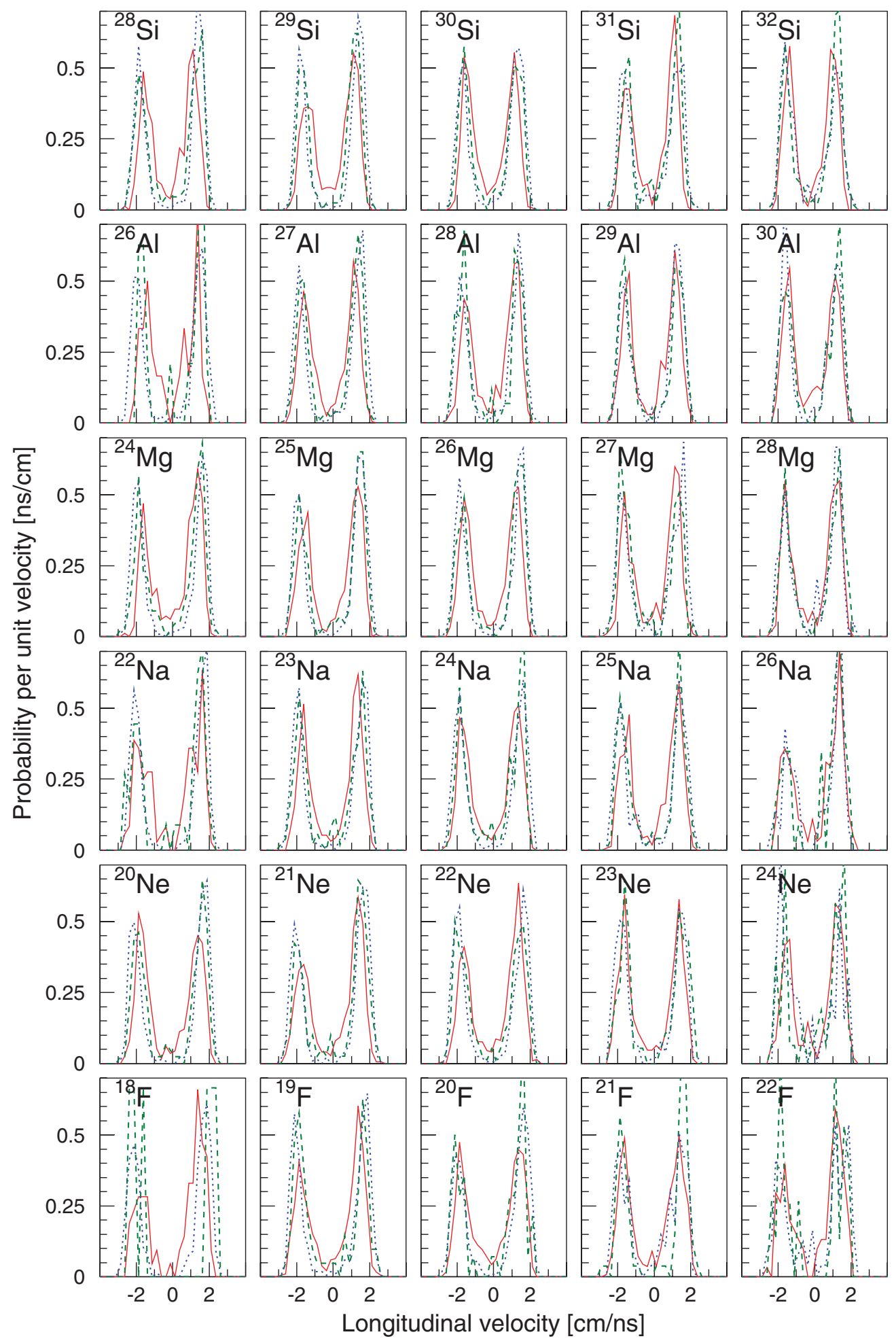

FIG. 18. (Color online) Same as Fig. 17 but for $9 \leqslant Z \leqslant 14$.

accounts for the exact propagation of the hot multifragmentation products in their mutual Coulomb field and is, therefore, supposed to yield reasonable predictions of the asymptotic velocities, if the initial conditions are realistic. Figure 9 demonstrates that this mechanism does not yield single-peaked LVDs. Even many-body $(\geqslant 3)$ breakup configurations (dark red component) bear clear signs of Coulomb repulsion. This suggests that most of the breakup configurations must be quasibinary, with one or two large fragments completely dominating the Coulomb dynamics, possibly accompanied by nucleons and very light-charged particles. Note that ABLA07's multifragmentation mechanism seems to produce flatter 

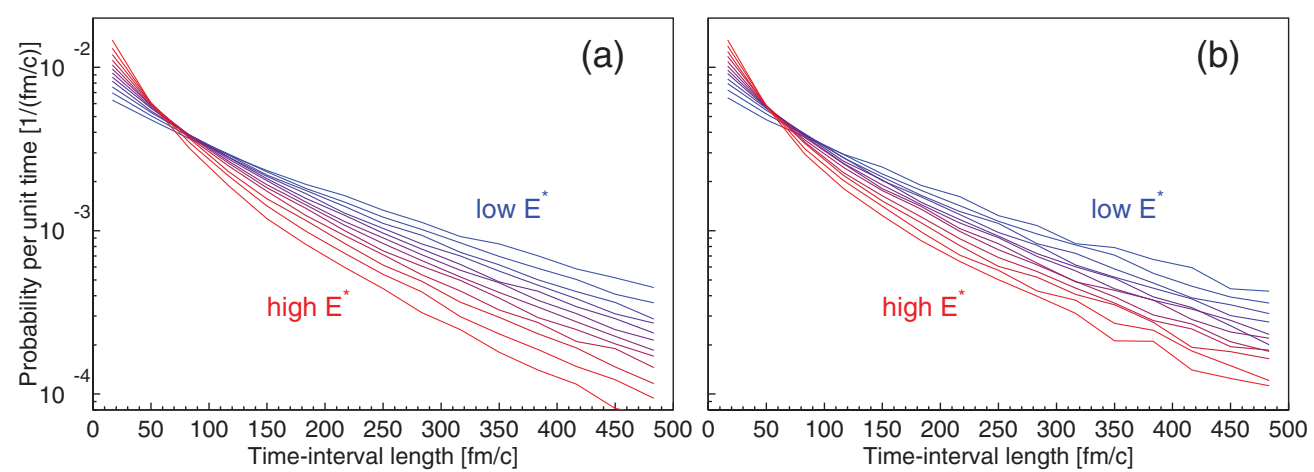

FIG. 19. (Color online) Distributions of time intervals between asymmetric splits. Curve colors, from blue to red, correspond to increasing values of the excitation energy. Each curve is normalized to 1 . (a) $p+{ }^{56} \mathrm{Fe}$; (b) $p+{ }^{136} \mathrm{Xe}$.

distributions, although its cross section is largely insufficient to explain the experimental shapes.

Finally, we comment briefly about the contribution of dynamical emission of cascade clusters to the LVDs. We remind the reader that INCL 4.5 by default produces clusters through a coalescence mechanism up to $A=8$ included [12]. Indeed, Figs. 8 and 9 show a cascade contribution for the lightest IMFs. Cascade clusters, which are high-energy particles in direct kinematics, constitute an asymmetric tail that extends in the backward direction in inverse kinematics. The cascade-cluster tail is sometimes responsible for a large forward-backward asymmetry of the LVD. A similar signature was observed in the ${ }^{136} \mathrm{Xe}+{ }^{1} \mathrm{H}$ data set [10] but was attributed to fluctuations in the recoil momentum of the cascade remnant. The INCL4.5 model suggests a different interpretation.

\section{TIME INTERVAL BETWEEN FRAGMENT EMISSIONS}

We have so far considered residue-production cross sections, correlations among de-excitation products, and distributions of longitudinal velocity of the emitted fragments. For most of these end points, the INCL4.5/GEMINI++ model provided the most accurate description of the experimental data (with the exception of the $p+{ }^{136}$ Xe LVDs, which no model seems to be able to reproduce). This result can be taken as evidence that a multifragmentation model is unnecessary to describe the reactions studied in the present paper. The solidity of this argument, however, relies on the internal consistency of the application of the INCL4.5/GEMINI++ model to the systems considered. We will now proceed to show that the time interval between fragment emissions for highly excited cascade remnants becomes comparable to the typical multifragmentation time scale.

The GEMINI++ model keeps an internal clock of the decay process, which is readily available to the user. For a compound nucleus with decay width $\Gamma$, the decay time is sampled from an exponential distribution with time constant $\hbar / \Gamma$. Thus, we select events with two or more asymmetric splits (two-split events) and compute the interval length $\Delta t_{\text {split }}$ between the earliest and the second-earliest emission. Note that this definition is the event-based equivalent of the " $2+$ asymmetric splits" particle classification above (Sec. IV A). If more than two splits occur during an event, we consider only the two earliest; the idea is that we are interested in short intervals and emissions become more separated in time as the excitation energy is evacuated. Note that not every asymmetric split leads to observed fragments; if the excitation energy of the emitted fragment is sufficient, it can completely disassemble in light-charged particles and remain unobserved. Thus, two asymmetric splits do not necessarily correspond to three observed fragments.

We then define 12 bins in excitation energy per nucleon by requiring that two-split events be uniformly partitioned over the bins. Since there are only few two-split events at low excitation energy, the first bin is very broad (from zero to $4.2 A \mathrm{MeV}$ for ${ }^{56} \mathrm{Fe}$ and $2.8 A \mathrm{MeV}$ for ${ }^{136} \mathrm{Xe}$ ). Finally, for each bin we construct a distribution of interval lengths. This procedure permits studying how time intervals evolve as a function of the excitation energy of the cascade remnant.

Figure 19 displays the distributions of time intervals for the 12 excitation-energy bins. The lowest excitation-energy bins are given above. The highest excitation-energy bins range from $8.8 A \mathrm{MeV}\left({ }^{56} \mathrm{Fe}\right)$ and $4.6 A \mathrm{MeV}\left({ }^{136} \mathrm{Xe}\right)$ to infinity. One notices that the distributions corresponding to low excitation energies are close to exponential. All distributions are very broad, with heavy tails extending up to several thousands $\mathrm{fm} / c$; for this reason, it is inappropriate to characterize the distributions using their mean and/or their standard deviation. In what follows, we will rather rely on the median and the interquartile range, which are more robust.

The dotted lines in Fig. 20 represent the energy-differential cross section for two-split events (right scale). One immediately notices that two-split events are concentrated at higher excitation energies per nucleon in ${ }^{56} \mathrm{Fe}$ than in ${ }^{136} \mathrm{Xe}$. The ${ }^{56} \mathrm{Fe}$ distribution extends up to very high excitation energies, comparable to or larger than the total binding energy of the remnant. Such remnants are rare but nevertheless possible. Interestingly, the maxima of the two-split cross sections are located at about $5.8\left({ }^{56} \mathrm{Fe}\right)$ and $3.6 \mathrm{~A} \mathrm{MeV}\left({ }^{136} \mathrm{Xe}\right)$, which are similar to ABLA07's multifragmentation thresholds cited in Sec. IV A. This strengthens the (perhaps coincidental) similarity between ABLA07's multifragmentation and GEMINI++'s two-split events that was observed in Sec. IV A.

On the left scale of Fig. 20 we report the medians (solid lines) and quartiles (dashed) of the interval-length distributions in each excitation-energy bin. The fact that the quartile curves 


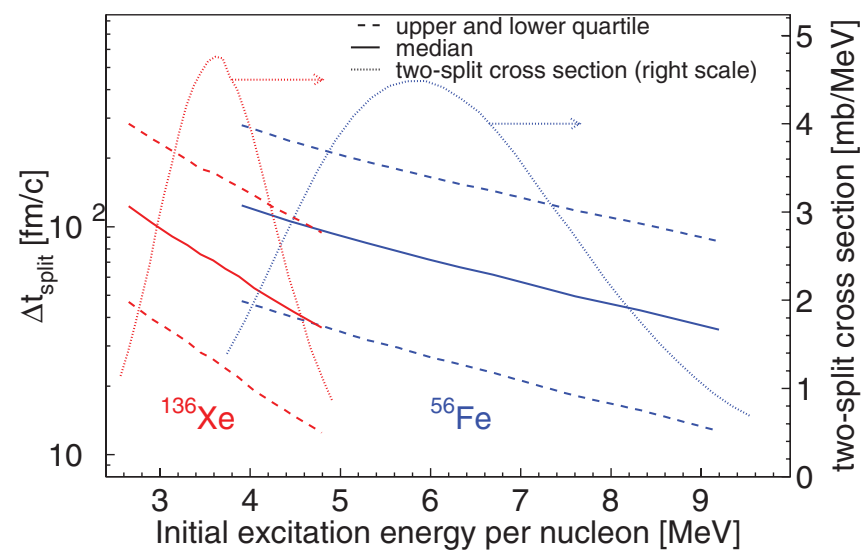

FIG. 20. (Color online) Median (solid lines) and quartiles (dashed lines) of the distributions of time-interval lengths between fragment emissions $\left(\Delta t_{\text {split }}\right)$ as a function of the initial excitation energy per nucleon. The model is INCL4.5/GEMINI++. Red (blue) lines correspond to the $p+{ }^{136} \mathrm{Xe}\left(p+{ }^{56} \mathrm{Fe}\right)$ reaction. Cross sections for two-split events are superimposed as dotted lines (right scale).

are approximately parallel to the median curve indicates that, up to a scale factor (note the logarithmic scale on the $\Delta t_{\text {split }}$ axis), the shapes of the distributions are approximately the same in all the bins. If we take the median as a measure of location, we can observe that typical interval lengths decrease as the initial excitation energy increases. Note also that, for the same excitation energy per nucleon, interval lengths are larger for ${ }^{56} \mathrm{Fe}$ than for ${ }^{136} \mathrm{Xe}$.

INCL4.5/GEMINI++ thus predicts that typical intervals between fragment emissions last a few hundred $\mathrm{fm} / c$ at the onset and reach $70-75 \mathrm{fm} / c$ at the peak of the two-split cross section (independently of the target). For higher excitation energies, even shorter times are expected. These numbers are comparable to the typical multifragmentation time scale of a few tens of $\mathrm{fm} / \mathrm{c}$ [31,32], suggesting a continuous transition between sequential binary decay at low energy and the expected multifragmentation regime at high energy. This aspect suggests that a binary-decay model can generate final states that are similar to those that a multifragmentation model would produce.

The time interval between fragment emissions represents an upper bound for the interval between any two consecutive binary decays. As the excitation energy increases, this time eventually becomes comparable to the relaxation time of the system. Under these conditions it is difficult to justify the compound-nucleus hypothesis, which assumes a completely equilibrated system. However, it is difficult to provide quantitatively accurate estimates of the relaxation time of a highly excited nuclear system. Even if we disregard the question of equilibration, however, the asymptotic (observable) escape velocities of the emitted charged particles are sensitive to the length of the interval between emissions [31] and should, in principle, be determined from the solution of the equations of motion of the emitted fragments in their mutual Coulomb field. In GEMINI++, as in most statistical deexcitation codes (including ABLA07 and even SMM's secondary deexcitation phase), it is assumed that decay products have already attained their asymptotic velocity before they undergo any subsequent decay. However, Coulomb interactions among closely emitted fragments will distort their kinematical correlations. The importance of an exact solution of the equations of motion could be evaluated by studying observables that are sensitive to the deexcitation kinematics, such as LVDs. Note, however, that SMM does include a numerical solver for the Coulomb trajectories of the hot fragments, but it is still unable to reproduce the experimental LVDs for $p+{ }^{136} \mathrm{Xe}$.

\section{CONCLUSIONS}

We have used the tools of coupled intranuclear-cascade and statistical-deexcitation models to search the $1-\mathrm{GeV} p+{ }^{56} \mathrm{Fe}$ and $p+{ }^{136} \mathrm{Xe}$ reactions for signatures of multifragmentation. The choice of the cascade model has some influence on the distributions of remnant size and excitation energy; in particular, dynamical emission of clusters during the cascade stage has a sensible influence on the remnant-mass distribution and, thus, on the residue-production cross sections. This leads to a rather large sensitivity of calculated residue-production cross sections on the cascade model. For the purpose of this study, we chose to fix the cascade model by requiring that it correctly reproduce residue-production cross sections close to the target nuclide, which are typically understood as due to the evaporation of lowly excited cascade remnants and are rather insensitive to the choice of the deexcitation model.

Calculations indicate that the inclusion of a multifragmentation stage is not crucial for adequate prediction of residue-production cross sections. We, thus, confirm the insensitiveness of this observable to the deexcitation mechanism. However, different deexcitation models propose widely different reconstructions of the residue-production cross sections in terms of elementary processes, suggesting that semiexclusive observables can help discriminate among different deexcitation mechanisms. Comparisons with measured fragmenthelium correlations, $Z_{1}-Z_{2}$ distributions, and IMF-gated $Z_{\text {bound }}$ distributions [6] favor binary de-excitation models such as GEMINI++ or models predicting very small multifragmentation cross sections such as ABLA07. We conclude that a multifragmentation model is not necessary for the description of inclusive and semiexclusive observables. This does not mean that the presence of multifragmentation is ruled out in these reactions but rather that binary decay can generate final states similar to those produced by multifragmentation models, at least close to the multifragmentation threshold.

Somewhat ambiguous conclusions can be drawn from the qualitative study of longitudinal-velocity distributions. Contrary to previous claims [9], we find that pure binary decay can account for the distributions measured in $p+{ }^{56} \mathrm{Fe}$. The observed single-peaked component can be ascribed to multifragmentation or deexcitation residues, or both, depending on the deexcitation model considered. On the other hand, none of the considered deexcitation models can explain the existence of the observed single-peaked component in $p+{ }^{136} \mathrm{Xe}$. Even numerical integration of the Coulomb trajectories of the multifragmentation products, as implemented in SMM, predicts double-peaked longitudinal-velocity distributions. Therefore, the shape of the longitudinal-velocity distribution is an ambiguous signature of the deexcitation mechanism. 
The INCL4.5/GEMINI + calculations suggest that $p+{ }^{136} \mathrm{Xe}$ residues with $4 \leqslant Z \lesssim 40$ are mostly produced in events with one asymmetric split, with no contribution from de-excitation residues; it is far from obvious that a similar mechanism can produce single-peaked longitudinal-velocity distributions. If the single-peaked component in $p+{ }^{136} \mathrm{Xe}$ must be ascribed to multifragmentation, we would expect the multifragmentation signature to be even more visible in $p+{ }^{56} \mathrm{Fe}$, but that does not appear to be the case.

We have also studied the time interval between asymmetric splits in INCL4.5/GEMINI++. At the highest excitation energies per nucleon, the model predicts interval lengths comparable with the typical multifragmentation time scale. This, again, suggests a smooth transition between the binary-decay and the multifragmentation regimes and illustrates how binary decay can generate multi-fragmentation-like final states, as mentioned above. It is not clear whether equilibration times of the order of the time interval between asymmetric splits are sufficiently long to justify GEMINI++'s compound-nucleus hypothesis; in any case, closely packed binary emissions of charged fragments are expected to distort the asymptotic
Coulomb velocities. This effect is not accounted for in any of the models considered in the present work.

In conclusion, binary decay yields a satisfactory description of most of the observables considered in this paper. The application of binary-decay models to cascade remnants with very large excitation energies generates final states that resemble those produced by multifragmentation models. The good agreement of INCL4.5/GEMINI++ and INCL4.5/ABLA07 with the experimental data considered in this paper probably indicates that events with very high excitation energy per nucleon do not significantly contribute to the studied observables.

\section{ACKNOWLEDGMENTS}

We thank the authors of the deexcitation models (A. Botvina, R. J. Charity, A. Kelić-Heil, M. V. Ricciardi, and K.-H. Schmidt) for enlightening discussions. P. Kaitaniemi's help for the GEANT4 simulation of the SPALADIN setup is gratefully acknowledged.
[1] Various authors, Eur. Phys. J. A 30 (2006).

[2] H. Aït Abderrahim, P. Baeten, D. D. Bruyn, J. Heyse, P. Schuurmans, and J. Wagemans, Nucl. Phys. News 20, 24 (2010).

[3] M. Durante, Riv. Nuovo Cimento 25, 1 (2002).

[4] Shielding Aspects of Accelerators, Targets and Irradiation Facilities: SATIF 10 Workshop Proceedings (OECD, Geneva, Switzerland, 2011).

[5] J.-C. David et al., in Shielding Aspects of Accelerators, Targets and Irradiation Facilities: SATIF 10 Workshop Proceedings (OECD, Geneva, Switzerland, 2011), p. 273; S. Leray, J.-C. David, M. Khandaker, G. Mank, A. Mengoni, N. Otsuka, D. Filges, F. Gallmeier, A. Konobeyev, and R. Michel, J. Korean Phys. Soc. 59, 791 (2011); "IAEA benchmark of spallation models," official web site, [http://www-nds.iaea.org/spallations]

[6] E. Le Gentil et al., Phys. Rev. Lett. 100, 022701 (2008).

[7] C. Villagrasa-Canton et al., Phys. Rev. C 75, 044603 (2007).

[8] P. Napolitani et al., Phys. Rev. C 76, 064609 (2007).

[9] P. Napolitani, K.-H. Schmidt, A. S. Botvina, F. Rejmund, L. Tassan-Got, and C. Villagrasa, Phys. Rev. C 70, 054607 (2004).

[10] P. Napolitani, K.-H. Schmidt, and L. Tassan-Got, J. Phys. G: Nucl. Part. Phys. 38, 115006 (2011).

[11] A. Boudard, J. Cugnon, S. Leray, and C. Volant, Phys. Rev. C 66, 044615 (2002).

[12] J. Cugnon, A. Boudard, S. Leray, and D. Mancusi, J. Korean Phys. Soc. 59, 955 (2011).

[13] Y. Yariv and Z. Fraenkel, Phys. Rev. C 20, 2227 (1979); 24, 488 (1981).

[14] A. Kelić, M. V. Ricciardi, and K.-H. Schmidt, in Joint ICTPIAEA Advanced Workshop on Model Codes for Spallation Reactions (IAEA, Trieste, Italy, 2008), p. 181.
[15] J. B. Natowitz, R. Wada, K. Hagel, T. Keutgen, M. Murray, A. Makeev, L. Qin, P. Smith, and C. Hamilton, Phys. Rev. C 65, 034618 (2002).

[16] V. F. Weisskopf and D. H. Ewing, Phys. Rev. 57, 472 (1940).

[17] R. J. Charity, in Joint ICTP-IAEA Advanced Workshop on Model Codes for Spallation Reactions (IAEA, Trieste, Italy, 2008), p. 139.

[18] W. Hauser and H. Feshbach, Phys. Rev. 87, 366 (1952).

[19] L. G. Moretto, Nucl. Phys. A 247, 211 (1975).

[20] A. J. Sierk, Phys. Rev. Lett. 55, 582 (1985).

[21] D. Mancusi, R. J. Charity, and J. Cugnon, Phys. Rev. C 82, 044610 (2010).

[22] D. Mancusi, R. J. Charity, and J. Cugnon, EPJ Web of Conferences 17, 10003 (2011).

[23] J. P. Bondorf, A. S. Botvina, A. S. Iljinov, I. N. Mishustin, and K. Sneppen, Phys. Rep. 257, 133 (1995).

[24] A. S. Botvina, in Joint ICTP-IAEA Advanced Workshop on Model Codes for Spallation Reactions (IAEA, Trieste, Italy, 2008), p. 131.

[25] N. Bohr and J. A. Wheeler, Phys. Rev. 56, 426 (1939).

[26] A. Boudard, J. Cugnon, S. Leray, and C. Volant, Nucl. Phys. A 740, 195 (2004).

[27] S. Furihata, Nucl. Instrum. Methods B 171, 251 (2000).

[28] A. V. Ignatyuk, G. N. Smirenkin, and A. S. Tishin, Sov. J. Nucl. Phys. 21, 255 (1975).

[29] T. Gorbinet, Ph.D. thesis, Université Paris-Sud 11, Paris, France, 2011.

[30] E. Le Gentil, Ph.D. thesis, Université d'Evry, France, 2006.

[31] V. E. Viola et al. Phys. Rep. 434, 1 (2006).

[32] B. Borderie and M. F. Rivet, Prog. Part. Nucl. Phys. 61, 551 (2008). 